\title{
High energy cosmic ray physics with underground muons in MACRO. II. Primary spectra and composition
}

M. Ambrosio, ${ }^{12}$ R. Antolini, ${ }^{7}$ G. Auriemma,${ }^{14, *}$ R. Baker, ${ }^{11}$ A. Baldini,${ }^{13}$ G. C. Barbarino, ${ }^{12}$ B. C. Barish, ${ }^{4}$ G. Battistoni,${ }^{6, \dagger}$ R. Bellotti, ${ }^{1}$ C. Bemporad ${ }^{13}$ P. Bernardini,${ }^{10}$ H. Bilokon, ${ }^{6}$ V. Bisi,${ }^{16}$ C. Bloise, ${ }^{6}$ T. Bosio, ${ }^{7}$ C. Bower,${ }^{8}$ S. Bussino, ${ }^{14}$ F. Cafagna, ${ }^{1}$ M. Calicchio, ${ }^{1}$ D. Campana,${ }^{12}$ M. Carboni,${ }^{6}$ M. Castellano, ${ }^{1}$ S. Cecchini, ${ }^{2, \$}$ F. Cei,${ }^{13,8}$ V. Chiarella, ${ }^{6}$ A. Corona,${ }^{14}$ S. Coutu, ${ }^{11}$ G. De Cataldo,${ }^{1}$ H. Dekhissi ${ }^{2, \|}$ C. De Marzo, ${ }^{1}$ I. De Mitri, ${ }^{9}$ M. De Vincenzi, ${ }^{14, \pi}$ A. Di Credico, ${ }^{7}$ O. Erriquez, ${ }^{1}$ R. Fantini, ${ }^{2}$ C. Favuzzi, ${ }^{1}$ C. Forti, ${ }^{6}$ P. Fusco, ${ }^{1}$ G. Giacomelli, ${ }^{2}$ G. Giannini, ${ }^{13,}{ }^{* *}$ N. Giglietto, ${ }^{1}$ M. Goretti, ${ }^{14,4}$ M. Grassi, ${ }^{13}$ A. Grillo, ${ }^{7}$ F. Guarino, ${ }^{12}$ P. Guarnaccia,,${ }^{1}$ C. Gustavino, ${ }^{7}$ A. Habig, ${ }^{8}$ K. Hanson,,${ }^{11}$ A. Hawthorne,${ }^{8}$ R. Heinz,${ }^{8}$ J. T. Hong, ${ }^{3}$ E. Iarocci, ${ }^{6, \dagger}$ E. Katsavounidis, ${ }^{4}$ E. Kearns, ${ }^{3}$ S. Kyriazopoulou, ${ }^{4}$ E. Lamanna, ${ }^{14}$ C. Lane, ${ }^{5}$ D. S. Levin, ${ }^{11}$ P. Lipari, ${ }^{14}$ N. P. Longley, ${ }^{17}$ M. J. Longo, ${ }^{11}$ G. Mancarella, ${ }^{10}$ G. Mandrioli, ${ }^{2}$ A. Margiotta-Neri, ${ }^{2}$ A. Marini, ${ }^{6}$ D. Martello, ${ }^{10}$ A. Marzari-Chiesa ${ }^{16}$ M. N. Mazziotta, ${ }^{1}$ D. G. Michael, ${ }^{4}$ S. Mikheyev, ${ }^{7, \neq}$ L. Miller, ${ }^{8}$ P. Monacelli, ${ }^{9}$ T. Montaruli, ${ }^{1}$ M. Monteno, ${ }^{16}$ S. Mufson, ${ }^{8}$ J. Musser,${ }^{8}$ D. Nicoló,,${ }^{13,}$ R. Nolty, ${ }^{4}$ C. Okada, ${ }^{3}$ C. Orth ${ }^{3}$ G. Osteria, ${ }^{12}$ O. Palamara, ${ }^{10}$ S. Parlati, ${ }^{7}$ V. Patera,,${ }^{6} \dagger$ L. Patrizii, ${ }^{2}$ R. Pazzi, ${ }^{13}$ C. W. Peck, ${ }^{4}$ S. Petrera, ${ }^{10}$ P. Pistilli, ${ }^{10}$ V. Popa,${ }^{2,8}$ A. Rainó ${ }^{1}$ J. Reynoldson, ${ }^{7}$ M. Ricciardi,${ }^{10}$ F. Ronga, ${ }^{6}$ U. Rubizzo, ${ }^{12}$ A. Sanzgiri, ${ }^{15}$ F. Sartogo,${ }^{14}$ C. Satriano, ${ }^{14, *}$ L. Satta ${ }^{6, \dagger}$ E. Scapparone, ${ }^{7}$ K. Scholberg, ${ }^{4}$ A. Sciubba,${ }^{6, \dagger}$ P. Serra-Lugaresi ${ }^{2}$ M. Severi, ${ }^{14}$ M. Sitta, ${ }^{16}$ P. Spinelli, ${ }^{1}$ M. Spinetti, ${ }^{6}$ M. Spurio, ${ }^{2}$ R. Steinberg, ${ }^{5}$ J. L. Stone, ${ }^{3}$ L. R. Sulak, ${ }^{3}$ A. Surdo, ${ }^{10}$ G. Tarlé, ${ }^{11}$ V. Togo, ${ }^{2}$ V. Valente, ${ }^{6}$ C. W. Walter, ${ }^{4}$ and R. Webb ${ }^{15}$

(MACRO Collaboration)

\author{
${ }^{1}$ Dipartimento di Fisica dell'Università di Bari and INFN, 70126 Bari, Italy \\ ${ }^{2}$ Dipartimento di Fisica dell'Università di Bologna and INFN, 40126 Bologna, Italy \\ ${ }^{3}$ Physics Department, Boston University, Boston, Massachusetts 02215 \\ ${ }^{4}$ California Institute of Technology, Pasadena, California 91125 \\ ${ }^{5}$ Department of Physics, Drexel University, Philadelphia, Pennsylvania 19104 \\ ${ }^{6}$ Laboratori Nazionali di Frascati dell'INFN, 00044 Frascati (Roma), Italy \\ ${ }^{7}$ Laboratori Nazionali del Gran Sasso dell'INFN, 67010 Assergi (L'Aquila), Italy \\ ${ }^{8}$ Departments of Physics and Astronomy, Indiana University, Bloomington, Indiana 47405 \\ ${ }^{9}$ Dipartimento di Fisica dell'Università dell'Aquila and INFN, 67100 L'Aquila, Italy \\ ${ }^{10}$ Dipartimento di Fisica dell'Università di Lecce and INFN, 73100 Lecce, Italy \\ ${ }^{11}$ Department of Physics, University of Michigan, Ann Arbor, Michigan 48109 \\ ${ }^{12}$ Dipartimento di Fisica dell'Università di Napoli and INFN, 80125 Napoli, Italy \\ ${ }^{13}$ Dipartimento di Fisica dell'Università di Pisa and INFN, 56010 Pisa, Italy \\ ${ }^{14}$ Dipartimento di Fisica dell'Università di Roma 'La Sapienza"' and INFN, 00185 Roma, Italy \\ ${ }^{15}$ Physics Department, Texas A\&M University, College Station, Texas 77843 \\ ${ }^{16}$ Dipartimento di Fisica Sperimentale dell'Università di Torino and INFN, 10125 Torino, Italy \\ 17 Swarthmore College, Swarthmore, Pennsylvania 19081
}

(Received 3 September 1996; revised manuscript received 5 February 1997)

\begin{abstract}
Multimuon data from the MACRO experiment at Gran Sasso have been analyzed using a new method, which allows one to estimate the primary cosmic ray fluxes. The estimated all-particle spectrum is higher and flatter than the one obtained from direct measurements but is consistent with EAS array measurements. The spectral indexes of the fitted energy spectrum are $2.56 \pm 0.05$ for $E<500 \mathrm{TeV}$ and $2.9 \pm 0.3$ for $E>5000 \mathrm{TeV}$ with a gradual change at intermediate energies. The average mass number shows little dependence on the primary energy below $1000 \mathrm{TeV}$, with a value of $10.1 \pm 2.5$ at $100 \mathrm{TeV}$. At higher energies the best fit average mass shows a mild increase with energy, even though no definite conclusion can be reached taking into account errors. The fitted spectra cover a range from $\sim 50 \mathrm{TeV}$ up to several thousand TeV. [S0556-2821(97)00915-6]
\end{abstract}

PACS number(s): 13.85.Tp, 96.40.De, 96.40.Tv, 98.70.Sa

\footnotetext{
*Also at Università della Basilicata, 85100 Potenza, Italy.

${ }^{\dagger}$ Also at INFN Milano, 20133 Milano, Italy.

\#Also at Istituto TESRE/CNR, 40129 Bologna, Italy.

${ }^{\S}$ Also at Scuola Normale Superiore di Pisa, 56010 Pisa, Italy.

"Also at Faculty of Sciences, University Mohamed I, B.P. 424 Oujda, Morocco.

ITAlso Dipartimento di Fisica, Università di Roma Tre, Roma, Italy.
}

\footnotetext{
**Also at Università di Trieste and INFN, 34100 Trieste, Italy.

${ }^{\dagger}$ Also at Dipartimento di Energetica, Università di Roma, 00185 Roma, Italy.

$\sharp$ Also at Institute of Gravity and Space Sciences, 76900 Bucharest, Romania.

${ }_{\S}$ Also at Institute for Nuclear Research, Russian Academy of Science, 117312 Moscow, Russia.
} 


\section{INTRODUCTION}

A characteristic feature of the cosmic ray spectrum is the steepening that occurs between $10^{15}$ and $10^{16} \mathrm{eV}$, the "knee" of the spectrum. Several explanations of this phenomenon have been offered; many of these lead to substantial differences in the predicted spectrum and composition near the knee. At present, cosmic rays below about $10^{14} \times Z \mathrm{eV}$ are believed to be predominantly due to shock acceleration of particles by supernova blast waves in the interstellar medium [1]. At higher energies different mechanisms have been proposed, mainly involving acceleration from compact sources, like x-ray binaries or young supernova remnants [2]. More recently explosions of massive stars into their former stellar wind have been suggested as extensions of the basic supernova mechanism that could account for the whole knee region of cosmic ray spectrum [3]. Furthermore, the propagation and diffusion of cosmic rays in the Galaxy is generally considered to play a major role in the change of composition around the knee [2]. Therefore the interpretation of the knee is crucial in understanding the origin of the cosmic rays and may provide deeper insight into galactic acceleration and propagation phenomena.

Air shower arrays, air Cherenkov telescopes, and underground experiments can measure the whole region around the knee. In these indirect measurements the nature of the primary and its energy are inferred from the surface measurements of extensive air showers or from underground studies of the penetrating high energy muon component. Since the first observation of the knee [4] several experiments have measured the primary spectrum [5-10] and confirmed its general features. However the uncertainties in the conversion of the observed shower parameters (e.g., shower size, muon size, etc.) to primary energy and in the normalization of the spectrum make it difficult to extract precise features of the spectrum and composition.

Underground experiments study primary composition comparing the measured muon multiplicity distribution with that calculated using trial models of the primary spectrum and composition. Following this approach composition studies have been carried out by Baksan [11], Homestake [12], NUSEX [13], Soudan [14], and MACRO [15-17]. MACRO composition analysis showed that experimental data are inconsistent, at high multiplicities, with the predictions of an asymptotically Fe-dominated composition, like the Heavy composition [18], and favor a lighter model. MACRO data are reasonably explained by models with flat or slowly increasing $\langle A\rangle$ as a function of the primary energy, as with the Light [18], CMC [19], and $\Sigma$ [20] models, except for the absolute rates, where the simulations are $\sim 25 \%$ low. A similar analysis has been performed on MACRO data in coincidence with the EAS-TOP array detector [20].

In the present analysis we describe an approach for measuring the primary spectrum and composition, using a multiparametric fit of the MACRO data. For this purpose we do not make any assumption based on specific theories of origin, acceleration or propagation of cosmic rays, but solely assume that elemental spectra can be expressed by simple power law functions. The calculation of the underground muon rates is done in the context of widely used hadronic interaction models. This approach allows us to obtain the model of the chemical composition which gives the best fit of multimuon data in the primary energy range between $10^{14}$ and $10^{17} \mathrm{eV}$, which is accessed by MACRO. A wide energy interval of about one decade below the knee is available where direct measurements and underground measurements of the primary cosmic ray composition overlap. In our fitting procedure data from direct experiments are used as starting points to constrain the composition below the knee.

The fitting procedure has been applied to a data sample of $\sim 4.4 \times 10^{6}$ muon events, of which $\sim 263000$ are multiple muons. This corresponds to a total live time of $5850 \mathrm{~h}$. Data selection, event analysis, and experimental method are described in detail in a companion paper [21].

In the next section we describe the details of the Monte Carlo simulation of multiple muons and analyze the possible sources of systematic uncertainties in our simulation. The third section presents the multiparametric fitting procedure. In Secs. IV and V we give the primary spectrum and composition arising from the fitting procedure. In Sec. VI the MACRO experimental data on muon decoherence and vertical muon intensity are compared with the predictions of the fitted composition model. Section VII discusses results and systematic uncertainties. Conclusions are given in Sec. VIII. Appendixes address technical features of the adopted best fit method.

\section{MONTE CARLO SIMULATION}

The interpretation of deep underground muon data requires a simulation which includes a hadronic interaction model, the air shower development, the propagation of muons through the rock and a detailed description of the detector.

The procedure to get usable Monte Carlo predictions is complex and heavily CPU time consuming. In our analysis the following simulation strategy has been adopted. In the event generation, after sampling mass, energy and direction of the primary particle, event by event, the relevant kinematic variables are calculated for muons surviving underground. This event production pass covers a wide energy spectrum, from a few $\mathrm{TeV}$ up to $10^{5} \mathrm{TeV}$ and the full solid angle up to a zenith angle of $60^{\circ}$. The upper limit at $10^{5} \mathrm{TeV}$ is motivated by the fact that only a few events per year are expected in our data sample at higher energies. For each event the impact position of the shower axis is randomized over an area much larger than that of the detector. If at least a segment of track enters the acceptance area of MACRO, a detailed simulation of detector and trigger is started. This simulation, based on the GEANT [22] code, includes a detailed description of all the known physics and detector effects (electromagnetic showering down to $500 \mathrm{keV}$, charge induction of the streamer signal onto the strips, electronic noise, etc.), and reproduces the experimental data at a satisfactory level of accuracy [21]. A final pass of the Monte Carlo sequence processes the simulated data through the standard MACRO analysis program to reconstruct muon tracks and other related quantities.

An important step of our fit analysis was the reduction of this huge amount of simulated data into suitable functions allowing a fast calculation of the muon rates. The muon rates at any detected multiplicity $N_{\mu}$ are given by 


$$
R\left(N_{\mu}\right)=\sum_{A} \int_{\Omega} d \Omega \int_{S} d S \int d E \Phi_{A}(E) \sum_{M_{\mu} \geqslant N_{\mu}} \mathcal{P}_{A}\left(E, \theta, \phi, M_{\mu}\right) \cdot \mathcal{D}\left(\left\{\vec{r}_{i}\right\}_{X_{0}} ; M_{\mu} \rightarrow N_{\mu}\right),
$$

where $E, A$, and $(\theta, \phi)$ denote the energy, mass number, and incident direction of the primary nucleus, whose spectrum is represented by the function $\Phi_{A}(E)$. $S$ represents the sampling area of the impact position of the shower axis, $X_{0}$, and $\Omega$ is the accepted solid angle. $\mathcal{P}_{A}\left(E, \theta, \phi, M_{\mu}\right)$ is the probability for a primary nucleus with physical parameters $E, A, \theta$, and $\phi$ to produce an event with $M_{\mu}$ muons at MACRO depth. $\mathcal{D}\left(\left\{\vec{r}_{i}\right\}_{X_{0}} ; M_{\mu} \rightarrow N_{\mu}\right)$ is the detector response term, giving the probability that a muon bundle, with $M_{\mu}$ muons located at $\left\{\vec{r}_{i}\right\}$ around the shower axis impact point $X_{0}$, be detected and reconstructed as an $N_{\mu}$ muon event. $\mathcal{P}_{A}\left(E, \theta, \phi, M_{\mu}\right)$ depends on the hadronic interaction model and muon propagation through the rock only. $\mathcal{D}\left(\left\{\overrightarrow{r_{i}}\right\}_{X_{0}} ; M_{\mu} \rightarrow N_{\mu}\right)$ depends on the muon bundle configuration and location inside the sampling area and on the detector geometry, acceptance, and overall reconstruction efficiency.

The sample of simulated data allows us to calculate the functions $D_{A}\left(E, N_{\mu}\right)$,

$$
D_{A}\left(E, N_{\mu}\right)=1 /(\Omega S) \int_{\Omega} d \Omega \int_{S} d S \sum_{M_{\mu} \geqslant N_{\mu}} \mathcal{P}_{A}\left(E, \theta, \phi, M_{\mu}\right) \cdot \mathcal{D}\left(\left\{\vec{r}_{i}\right\}_{X_{0}} ; M_{\mu} \rightarrow N_{\mu}\right),
$$

which represent the probabilities (averaged over $\Omega$ and $S$ ) for a primary of mass $A$ and energy per nucleus $E$ to be reconstructed as an event with $N_{\mu}$ muons in MACRO. Making use of these functions the muon rates are expressed by the energy convolution integral:

$$
R\left(N_{\mu}\right)=\Omega S \sum_{A} \int d E \Phi_{A}(E) \cdot D_{A}\left(E, N_{\mu}\right) .
$$

For this analysis, $\sim 400$ million events have been generated, subdivided in five $\log _{10}(E)$ bands, as follows: $3.8 \times 10^{8}$ primary nuclei $\left(1.1 \times 10^{6}\right.$ underground muon events) between 3 and $20 \mathrm{TeV}, 3.4 \times 10^{7}\left(1.7 \times 10^{6}\right)$ between 20 and $200 \mathrm{TeV}, 3.6 \times 10^{6}\left(1.1 \times 10^{6}\right)$ between 200 and 2000 $\mathrm{TeV}, 4.9 \times 10^{5}\left(4.3 \times 10^{5}\right)$ between 2000 and $20000 \mathrm{TeV}$ and $4.6 \times 10^{3}\left(4.3 \times 10^{3}\right)$ between 20000 and $100000 \mathrm{TeV}$. The CPU time increases with energy from $\sim 1 \mathrm{~s}$ MIPS/event ${ }^{1}$ in the lowest energy band up to $\sim 1000 \mathrm{~s}$ MIPS/event in the highest one. As a whole, one simulated $\mathrm{h}$ of MACRO livetime requires a CPU time of $\sim 380$ (MIPS h).

We assumed that primaries can be described using five mass groups $(H$, average mass $\langle A\rangle=1$; He, $\langle A\rangle=4$; CNO, $\langle A\rangle=14 ; \mathrm{Mg},\langle A\rangle=24 ; \mathrm{Fe},\langle A\rangle=56)$. The $D_{A}$ functions have been calculated as $25 \times 40\left(\log _{10}(E / \mathrm{GeV}), N_{\mu}\right)$ tables for each mass group, with $N_{\mu}$ ranging from 0 to 39 and $\log _{10}(E / \mathrm{GeV})$ from 3 to 8 . The choice of the sizes of these tables is of crucial importance in the fitting procedure we are going to describe in Sec. III. Our choice is a compromise of having a fine sampling of primary energies and muon multiplicities and a tolerable size of the simulated event sample. We established that this table size allows us to obtain, inserting the tabulated $D_{A}$ functions in Eq. (3), muon multiplicity distributions as accurate as from direct use of simulated events, for any fixed composition.

There are some systematic uncertainties in the Monte Carlo predictions that have been investigated in detail. These

\footnotetext{
${ }^{1}$ In our analysis we used computing machines of about 40 MIPS, 1 MIPS being assumed for HEMAS (see next section) running on VAX/780.
}

include uncertainties in the hadronic interaction model, in the knowledge of the map of the rock around MACRO, and muon propagation through the rock.

\section{A. Models of hadronic interactions}

The simulation of the hadronic interactions of primary cosmic rays with air nuclei plays an essential role in the interpretation of indirect cosmic ray data. The present analysis has been mainly based upon the HEMAS [23] shower code. It contains a hadron interaction event generator based on the parametrization of minimum bias events at the CERN Super Proton Synchrotron ( $\mathrm{Sp} \overline{\mathrm{p} S}$ ) collider by the UA5 experiment [24], generalized to nuclear targets. Multiplicity and pseudorapidity distributions reproduce collider data in the central region up to $\sqrt{s} \leqslant 900 \mathrm{GeV}$. Projectile diffraction is included. In the original version of this code, nuclear interaction is treated in the context of the pure superposition model. HEMAS is embedded in a shower program which follows the shower development in the atmosphere with an energy cut on secondary particles down to $0.5 \mathrm{TeV}$. It also includes code for three-dimensional muon propagation in the rock.

The results of this code have been parametrized [23] to provide a fast generator for underground muon physics. This parametrization has been used for the first analyses of MACRO experiment $[15,16,25,26]$, ignoring the correlation between muon multiplicity and lateral distribution. The need of a better comprehension of the underlying processes, and of understanding the associated systematics has lead to the use of the full code for the following MACRO analysis.

In the present analysis, a series of improvements have been implemented in the Monte Carlo simulation. The pure superposition model has been replaced with the more realistic semisuperposition model [27]. At a given energy, this causes larger fluctuations than those obtained with the superposition model, but the average values of the main observables remain the same. The effect of the geomagnetic field has been introduced for the high energy muons in the shower code, where the muon charge sign is randomly assigned. Charm hadroproduction has also been considered via inser- 


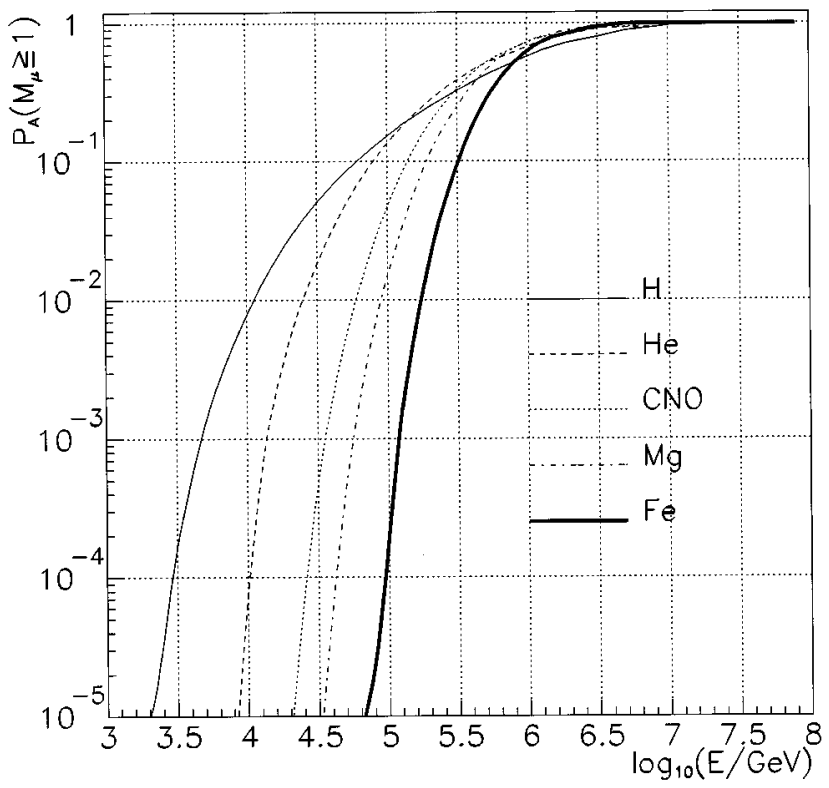

FIG. 1. Probabilities to give at least one muon at MACRO depth, calculated with HEMAS code.

tion of a simple heavy flavor generator ${ }^{2}$ to the standard hadron interaction routine of the HEMAS code. The contribution of charmed events to underground muons turned out to be negligible (of the order of a percent or less) on muon rates and of the order of a few percent on muon pair separations. Similar results have been obtained in [29], using the DMPJET [30] hadronic interaction model, where charm production is included. For this reason the heavy flavor generator was excluded from this composition analysis.

Figure 1 shows the inclusive probabilities $P_{A}\left(E, M_{\mu} \geqslant 1\right)$ of having at least one detectable muon at MACRO, as a function of the primary energy, for the five elemental groups used in our simulation. $P_{A}\left(E, M_{\mu} \geqslant 1\right)$ is related to $\mathcal{P}_{A}\left(E, \theta, \phi, M_{\mu}\right)$ through the relationship

$$
P_{A}\left(E, M_{\mu} \geqslant 1\right)=1 / \Omega \int_{\Omega} d \Omega \sum_{M_{\mu} \geqslant 1} \mathcal{P}_{A}\left(E, \theta, \phi, M_{\mu}\right) .
$$

At least one muon underground has been required in this plot, and this determines the increasing low energy limit when the mass group changes from hydrogen to iron. This has the consequence that heavier primaries are ineffective at lower energies, full efficiencies being reached roughly in the

${ }^{2} c \bar{c}$ pairs have been assumed to be produced independently of each other (uncorrelated production) with a differential cross section:

$$
d^{2} \sigma / d x_{F} d p_{T}^{2} \propto\left(1-\left|x_{F}\right|\right)^{\alpha} \exp \left(-\beta p_{T}^{2}\right),
$$

with $\alpha=3$ and $\beta=1.1(\mathrm{GeV} / c)^{-2}$ for each kind of charmed hadron. The charm production cross section for protons on air nuclei has been assumed with a dependence on the squared c.m. energy of the type $0.36+\log _{10}\left(s / 80 \mathrm{GeV}^{2}\right) \mathrm{mb}$ [28]. This energy dependence is roughly consistent with the collection of available experimental data, taking into account the measured charm production $A$ dependence.
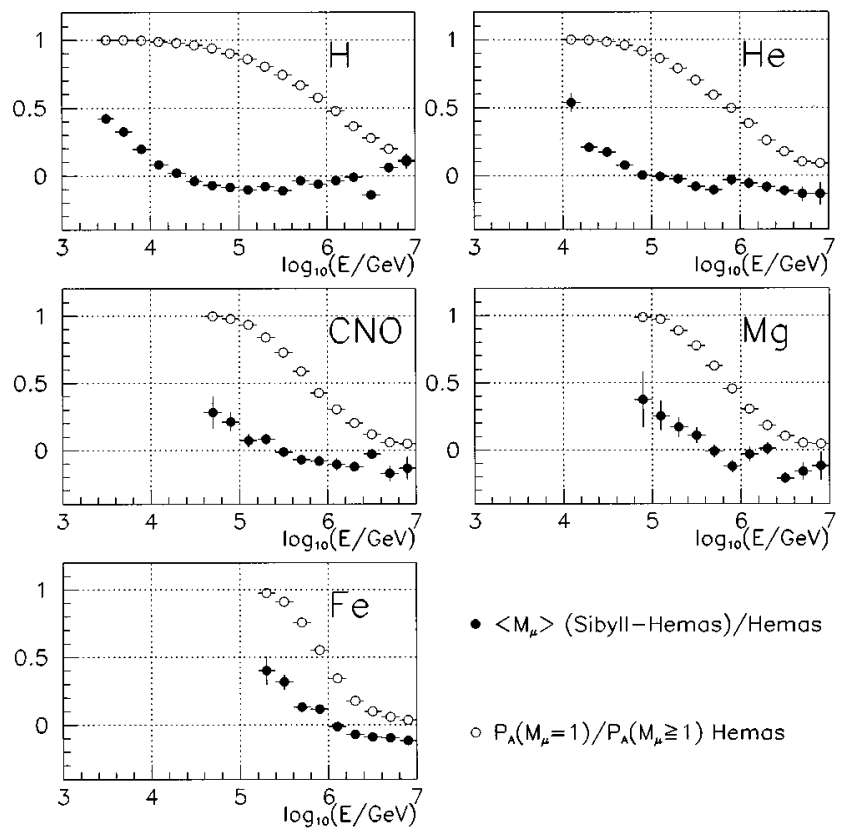

- $\left\langle\mathrm{M}_{\mu}\right\rangle$ (Sibyll-Hemas)/Hemas

o $P_{A}\left(M_{\mu}=1\right) / P_{A}\left(M_{\mu} \geqq 1\right)$ Hemas

FIG. 2. Full circles: relative differences between SIBYLL and HEMAS average muon multiplicities at MACRO: $(\mathrm{S}-\mathrm{H}) / \mathrm{H}$. Open circles: fraction of single muons as with HEMAS code.

knee region. Furthermore at these higher energies heavy elements are more prolific than protons in producing underground muons.

In order to estimate the dependence of our results on the adopted event generator, we have also used the SIBYLL interaction model [31]. This work has been done mostly for comparison on a reduced sample of simulated events. The SIBYLL event generator has been inserted in the same shower code already used for HEMAS. The SIBYLL model is more physically motivated than the HEMAS simulation, being based on the dual parton model [32] with the inclusion of hard processes such as minijet production [33]. In the future other models will be considered, such as the DPMJET code [30], which has a more complete treatment of nuclear effects than does SIBYLL.

Figure 2 shows, for each elemental group, the relative differences between SIBYLL and HEMAS average muon multiplicies at MACRO (full circles). It can be easily recognized that SIBYLL more effectively produces detectable muons near the underground muon production threshold, whereas at higher energies it approaches HEMAS. This behavior mainly affects the single muons (and the inclusive muon rates) since, at threshold, single muons dominate over multiple muons (open circles).

The multiplicity distributions of muons at MACRO depth have a similar behavior, with single muons slightly higher in SIBYLL and multiple muons slightly higher with HEMAS. Table I shows the relative differences between muon rates as obtained with SIBYLL and HEMAS for two different compositions ("light," proton rich and "heavy," iron rich [18]), at different $N_{\mu}$ intervals. The light and heavy compositions are extreme models: at increasingly higher energies the light composition contains a large proton component while the Heavy composition contains a large Fe component. Therefore, even with a drastic change in the composition spectra, the relative difference between the muon rates obtained in 
TABLE I. Relative differences between SIBYLL and HEMAS muon rates: $(\mathrm{S}-\mathrm{H}) / \mathrm{H}$. The values are given for the light and heavy compositions (see text).

\begin{tabular}{lcccc}
\hline \hline Composition & $M_{\mu}=1$ & $M_{\mu}=2-6$ & $M_{\mu}>6$ & $M_{\mu} \geqslant 1$ \\
\hline Light [18] & $0.058 \pm 0.003$ & $-0.08 \pm 0.01$ & $-0.07 \pm 0.05$ & $0.048 \pm 0.003$ \\
Heavy [18] & $0.072 \pm 0.004$ & $-0.03 \pm 0.01$ & $-0.02 \pm 0.02$ & $0.064 \pm 0.003$ \\
\hline
\end{tabular}

the framework of the two interaction models is always at most $\sim 7 \%$. It has to be noted that it mainly affects muon rates at low multiplicity, for which the statistical errors are very small. Comparative analyses $[34,31,35,36]$ among various underground muon features obtained with several hadronic interaction models (including HEMAS and SIBYLL) lead to similar results. Therefore we estimate the systematic uncertainty due to the hadronic interaction model to be of the order of $10 \%$, according to currently available models. Possible inadequacies of existing interaction models will be discussed futher in Sec. VII.

\section{B. Rock depth around MACRO}

The analysis of the vertical muon intensity measured in MACRO [21,37] has motivated a detailed study of the systematic uncertainties in the rock depth above MACRO. The rock depth $h$ (in m.w.e.) in each direction is obtained by multiplying the rock thickness by the average Gran Sasso rock density $\left(2.71 \pm 0.05 \mathrm{~g} / \mathrm{cm}^{3}\right)[21]$. The systematic uncertainties on $\mathrm{h}$ have two main sources: the uncertainties in the knowledge of the topographical map of the Gran Sasso region and in the interpolation procedure to transform it to a function of the zenith and azimuth angles; the assumption of a homogeneous mountain instead of a more realistically stratified structure [38].

The first term mainly causes point to point uncertainties of the order of few percent that marginally affect multimuon rates; the resulting systematic errors are somewhat less or at most equal to the statistical error at each muon multiplicity. The second term is mainly responsible for a possible systematic error on the absolute scale of the overall muon flux and is of the same size as the error of the average rock density (again few percent).

We have investigated the effect on the Monte Carlo predictions due to a net change of the rock depth by $-1 \%$, $-2 \%$, and $-5 \%$ everywhere, as originating from an average density uncertainty. The multimuon rates increase by $\sim+5 \%, \sim+10 \%$, and $\sim+25 \%$, respectively, roughly independently of the muon multiplicity. In other words, a net change of the rock depth, within the limits compatible with our rock uncertainties, affects the rates $R\left(N_{\mu}\right)$, but not the rates normalized to the singles, i.e., $r\left(N_{\mu}\right)=R\left(N_{\mu}\right) / R(1)$. To show this, in Fig. 3 the relative differences of $r\left(N_{\mu}\right)$, obtained with different average rock densities, with respect to the one at the Gran Sasso nominal density, are plotted as a function of the muon multiplicity. One can easily recognize that, within the statistical uncertainties of simulated data, $r\left(N_{\mu}\right)$ is roughly the same at any rock density and compatible with being independent of $N_{\mu}$. This fact reflects the loose but effective correlation between muon energy and primary energy, which is not considered when these effects are analyzed only using the muon survival probability.

\section{Muon propagation through the rock}

The original HEMAS code for the muon propagation through the rock (described in [23]) has been compared with the codes by Lipari and Stanev [39] and FLUKA [40]. The main differences between these codes and the original HEMAS muon propagation code is a more accurate treatment of the muon stochastic energy loss and of the radiative processes. Some theoretical uncertainties are still present anyway in the treatment of the radiative processes considered in the FLUKA and Lipari-Stanev codes. Moreover, uncertainties remain about the choice of the screening function used in the bremsstrahlung cross section.

Monte Carlo simulations show that while the uncertainties from the muon propagation affect the absolute muon rates at a level of $\sim 8 \%$ they do not affect the shape of multiplicity distributions.

\section{MULTIPARAMETRIC FIT OF THE COMPOSITION PARAMETERS}

In this analysis a minimization procedure is used to estimate the primary cosmic ray composition from the best fit of the MACRO experimental rates of multimuon events. Previous underground experiments have been able to perform only very simple fits (with only one free parameter) of experimental data $[13,12]$ in order to obtain information about primary composition. The main reason for that was the small detection area of their experimental apparata, so that only a small fraction of the muons in a high multiplicity event was

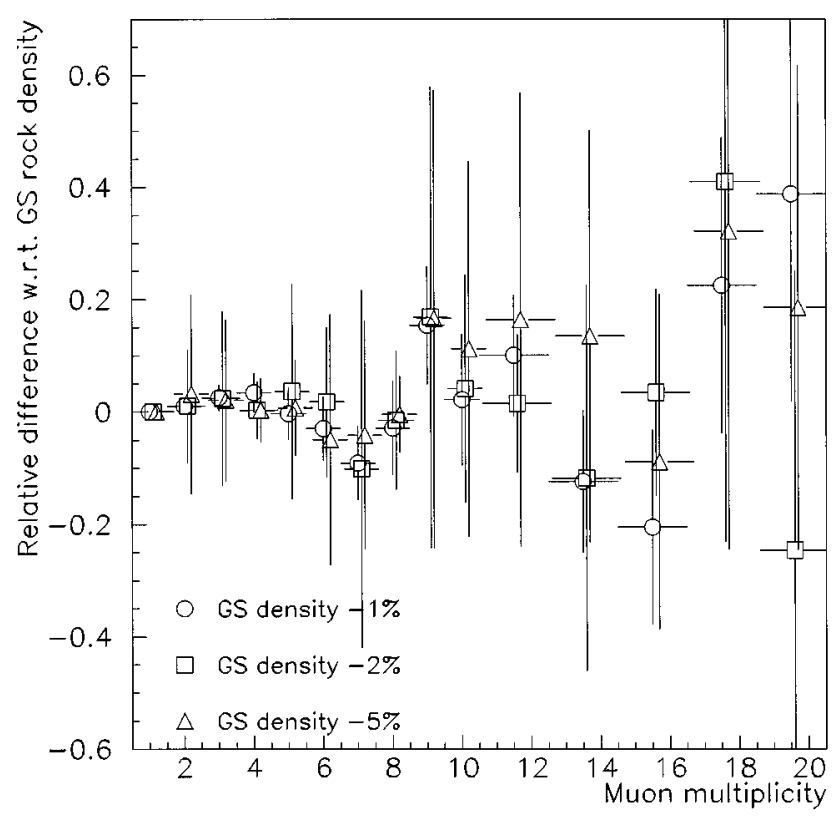

FIG. 3. Relative differences of $r\left(N_{\mu}\right)$ at different average rock densities with respect to the one at the Gran Sasso nominal density. 
seen by these detectors. On the contrary, the large acceptance of the MACRO detector is reflected in higher muon rates and sampling of very high multiplicities, up to largely separated muons.

In our fitting procedure, the predicted rates of events with $N_{\mu}$ reconstructed muons, $R\left(N_{\mu}\right)$, are calculated using formula (3). $\Phi_{A}(E)$ are the differential primary cosmic ray fluxes of the nuclei of mass $A$ that we aim to estimate, while the $D_{A}\left(E, N_{\mu}\right)$ functions are tabulated functions derived by our full Monte Carlo simulation. We assume that the energy spectrum of each elemental group can be expressed by simple power laws whose spectral indexes change at a mass dependent cutoff energy $E_{\text {cut }}(A)$ :

$$
\begin{array}{cc}
\Phi_{A}(E)=K_{1}(A) E^{-\gamma_{1}(A)} & \text { for } \quad E<E_{\mathrm{cut}}(A), \\
\Phi_{A}(E)=K_{2}(A) E^{-\gamma_{2}(A)} & \text { for } E>E_{\text {cut }}(A),
\end{array}
$$

with $K_{2}=K_{1} E_{\text {cut }}^{\gamma_{2}-\gamma_{1}}$. This corresponds to four free parameters $\left(K_{1}, \gamma_{1}, \gamma_{2}, E_{\text {cut }}\right)$ to be determined for each elemental primary spectrum that we want to estimate.

We minimize the function

$$
\chi_{M}^{2}=\sum_{N_{\mu}} \frac{\left[R^{\text {meas }}\left(N_{\mu}\right)-R\left(N_{\mu} \mid \text { parameters }\right)\right]^{2}}{\sigma^{2}\left[R^{\text {meas }}\left(N_{\mu}\right)\right]+\sigma^{2}\left[R\left(N_{\mu} \mid \text { parameters }\right)\right]},
$$

where $R^{\text {meas }}\left(N_{\mu}\right)$ are 39 experimental points of the MACRO multiplicity distribution [21], and $R\left(N_{\mu} \mid\right.$ parameters) are calculated using formula (3). The goal of this minimization procedure is to obtain a model of the chemical composition and elemental spectra of primary cosmic rays and an estimate of the errors on this model. The primary cosmic ray spectra are obtained from the estimate of the parameters at the minimum of the function (7). The errors on the spectra are calculated using the covariance matrix of the parameters.

The function minimization has been done using the MINUIT [41] package, a widely used application designed to compute the best fit parameter values and uncertainties, including correlations between the parameters.

\section{A. Preliminary tests}

Using five mass groups ( $\mathrm{H}, \mathrm{He}, \mathrm{CNO}, \mathrm{Mg}, \mathrm{Fe})$, we have 20 parameters to be fitted using formula (7). Such a high number of free parameters and the dependence of the muon rates on the composition parameters, through a convolution of primary spectra over a wide energy range, makes it difficult to estimate the primary composition using the procedure described above (see also Appendix A). Before going through the complexity of a minimization with so many parameters, we performed preliminary tests, assuming simplified hypotheses of primary compositions, which allow us to reduce the number of free parameters to be fitted. In particular, we checked if our multiplicity distribution was compatible with pure compositions or with compositions with only groups of elements.

With the assumption of a pure primary composition (only four free parameters), which is the simplest composition hypothesis that can be done, the minimization procedure does not converge for any primary mass. Therefore, our data rule
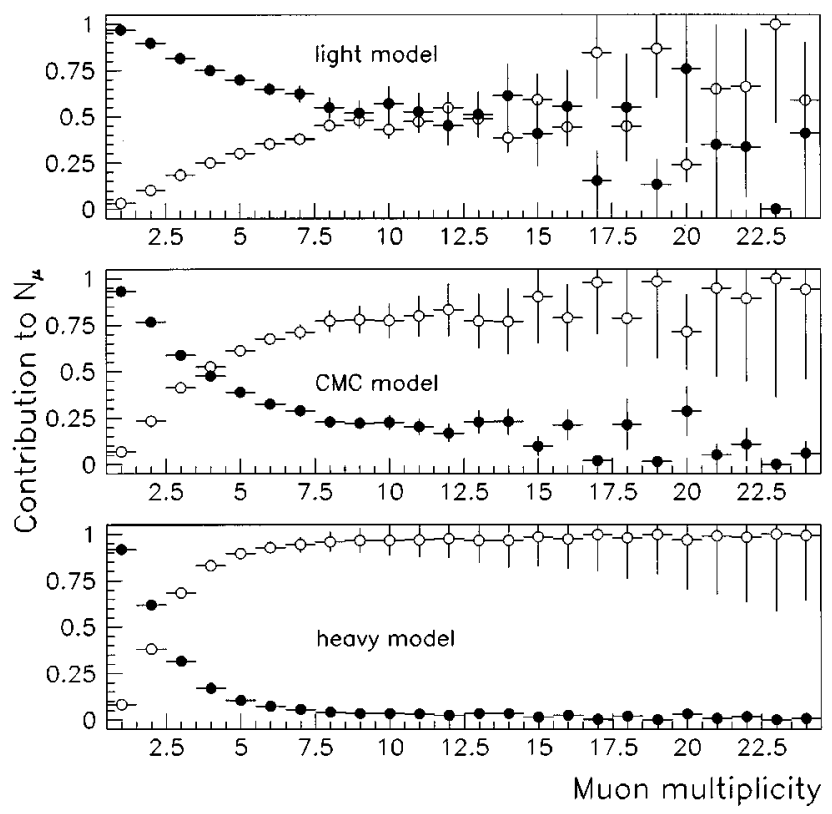

FIG. 4. Contribution of $(\mathrm{H}+\mathrm{He})$ (full circles) and $(\mathrm{CNO}+\mathrm{Mg}$ $+\mathrm{Fe}$ ) (open circles) groups to the multiplicity distribution for the light, $\mathrm{CMC}$, and heavy composition models.

out the hypothesis of pure compositions, such as extreme proton or iron pure compositions. Obviously a pure iron composition predicts too few low multiplicity events and too many high multiplicity events, whereas a pure proton composition can fit the integral muon rate properly, but predicts too few high multiplicity events.

The assumption of pure compositions is inadequate to represent the whole multiplicity distribution, since the different mass groups do not play the same role in the various multiplicity regions. Figure 4, which shows the contributions of the elemental groups to the multiplicity distribution calculated from the Monte Carlo simulation using different composition models, indicates that low multiplicity events mostly come from protons and helium nuclei, while high multiplicity events reflect much heavier primaries. Moreover, low multiplicity events come from primaries with energies less than few hundred $\mathrm{TeV}$ (below the knee), while high multiplicity events are produced by primaries in an energy region which includes the knee [16]. We therefore fitted the low multiplicity part $\left(N_{\mu}=1-6\right)$ of our experimental multiplicity distribution using only light $(\mathrm{H}+\mathrm{He})$ elements and assuming single power law energy spectra (corresponding to four free parameters). The remaining part of the distribution $\left(N_{\mu}>6\right)$ has been fitted independently with heavier mass groups $(\mathrm{CNO}+\mathrm{Mg}+\mathrm{Fe})$, assuming two power law spectra (corresponding to 12 free parameters). In both cases the multiparametric fitting procedure converges. The minimum $\chi_{M}^{2} / N_{\mathrm{DF}}$ is 1.7 for the low multiplicity fit and 0.5 for the high multiplicity fit. These results confirm qualitatively the dominance of different elemental groups at different multiplicities, but do not give acceptable spectra in the whole primary energy range. In fact, the loose correlation between muon multiplicity and primary energy prevents us from merging the spectra arising from the two separate fits.

\section{B. Results of the best fit procedure}

The simple preliminary tests described in the previous section show that a mixed composition with all the elemental 

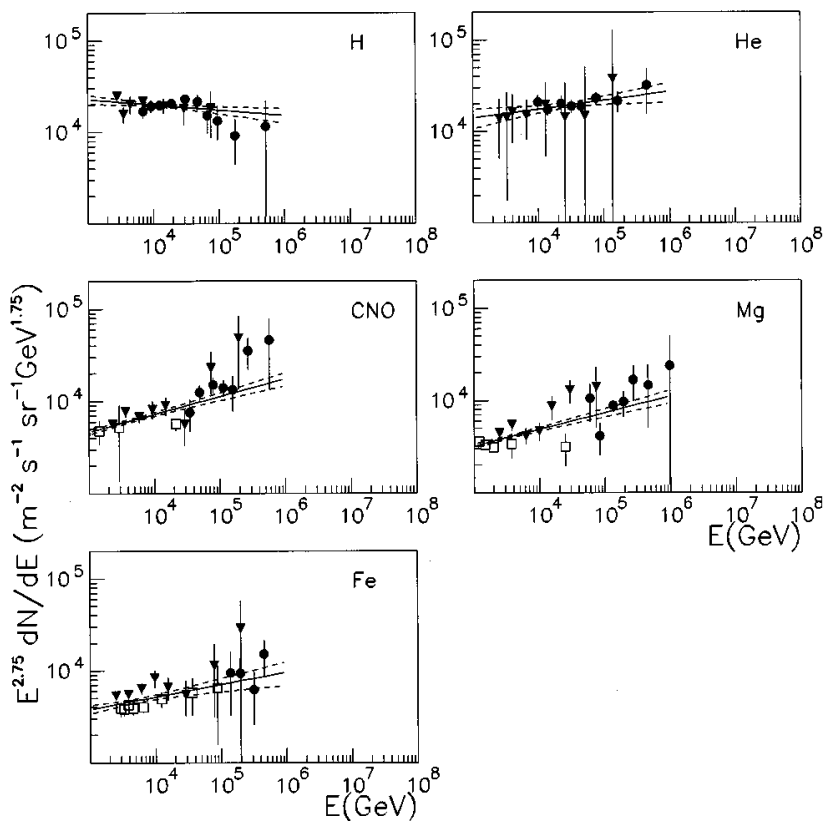

FIG. 5. Measurements of primary cosmic ray energy spectra from recent direct experiments. Full circles: JACEE [43], full triangles: SOKOL [44], open squares: CRN [45]. Primary spectra resulting from the fit of experimental points with single power law spectra are also shown (solid line: best fit value; dashed line $\pm 1 \sigma$ error). The $\chi^{2} / N_{\mathrm{DF}}$ are 14/15, 2.1/14, 26/17, 29/17, and 21/18 for $\mathrm{H}$, $\mathrm{He}, \mathrm{CNO}, \mathrm{Mg}$, and $\mathrm{Fe}$ mass groups, respectively.

groups is necessary to explain the experimental data in the whole multiplicity range of MACRO data. The ability of our best fit procedure to reconstruct primary compositions based on five elemental groups is described separately in Appendix E.

To get successful fits with five mass groups we are forced to reduce the number of free parameters. Constraints in the fit procedure, which reduce the number of free parameters, can be achieved by making physical assumptions on composition parameters. A wide class of composition models attributes the cutoff in the primary energy spectrum to particle leakage in the Galaxy at fixed magnetic rigidity [42]. We adopted this physical hypothesis in our fit, assuming that the energy cutoffs of elemental groups follow the relationship

$$
E_{\text {cut }}(Z)=E_{\text {cut }}(\mathrm{Fe}) \cdot Z / 26 \text {. }
$$

With this hypothesis the number of free parameters is 16 and the number of degree of freedom of $\chi_{M}^{2}$ is $\nu_{M}=23$.

Using this condition, the minimization of the $\chi_{M}^{2}$ function (7) is possible, if we impose suitable limits on the parameters to prevent them from taking on unphysical values because of numerical difficulties in the calculation. In order to bound the individual flux parameters below the knee [i.e., $\gamma_{1}(A)$ and $K_{1}(A)$ ], we have considered direct measurements from recent experiments (JACEE [43], SOKOL [44], and CRN [45]), whose data have been fitted with a single power law function [Eq. (5)]. The data we used as well as the fitted spectra are shown in Fig. 5. Each elemental group is well described by the assumed single power law function, giving an overall $\chi^{2} / N_{\mathrm{DF}}$ of $92 / 81 \simeq 1.1$. The fitted composition parameters are in good agreement with those obtained in a more complete analysis of cosmic ray direct data [46]. It is not possible to do the same exercise on the remaining parameters [i.e., $\gamma_{2}(A)$ and $\left.E_{\text {cut }}(\mathrm{Fe})\right]$, since individual fluxes are not measured above the knee and furthermore the knee energy cutoff is rather uncertain. Therefore the limits on these parameters have been inferred from all-particle EAS measurements. In summary, our boundary conditions have been defined as follows: $\gamma_{1}(A)$ and $K_{1}(A)$ within $\pm 5 \quad \sigma$ around their direct measurements fit best values, $\gamma_{2}(A)=2.8-3.2$, and $\log _{10}\left[E_{\text {cut }}(\mathrm{Fe}) / \mathrm{GeV}\right]=6-7.5$. With these conditions, the minimization of MACRO data points (7) is successful and the minimum is reached at $\chi_{M}^{2} / N_{\mathrm{DF}}=13 / 23 \simeq 0.6$ (hereafter referred to as the $M / A$ fit, $M / A$ standing for MACRO alone). The estimated parameters are given in Table VII. Unfortunately it was not possible to prevent some parameters from touching their boundary limits and then their errors are not meaningful. As explained in Appendix A, boundary conditions are generally undesired, since the parameters can be correctly estimated, but with unreliable errors due to numerical problems in the calculation of the covariance matrix.

For this reason we preferred to use a different approach. MACRO multimuon events are produced by primaries in the energy range of $\sim 50$ to $\sim 10^{5} \mathrm{TeV}$ [15-17]. A wide energy interval of about one decade below the knee is available where direct measurements and underground measurements overlap. Therefore we inserted direct measurements in the minimization function so that they can act as starting points and constrain the primary spectra below the knee. For this purpose the minimization function has been redefined as follows (see Appendix A):

$$
\xi^{2}=\lambda_{M} \chi_{M}^{2}+\lambda_{D} \chi_{D}^{2}
$$

where $\chi_{M}^{2}$ applies to MACRO multimuon data, as defined in Eq. (7), and $\chi_{D}^{2}$ applies to direct flux measurements:

$$
\chi_{D}^{2}=\sum_{A} \sum_{i=1}^{N_{A}} \frac{\left[\Phi_{A}^{\text {meas }}\left(E_{i}\right)-\Phi_{A}\left(E_{i} \mid \text { parameters }\right)\right]^{2}}{\sigma^{2}\left[\Phi_{A}^{\text {meas }}\left(E_{i}\right)\right]} .
$$

$\lambda_{M}$ and $\lambda_{D}$ are fixed weight parameters, and $\Phi_{A}\left(E_{i} \mid\right.$ parameters) are the primary cosmic ray fluxes of the nucleus $A$ at fixed primary energies $E_{i}$, defined by formula (5). In the $\chi_{D}^{2}$ term we used $91 \Phi_{A}^{\text {meas }}\left(E_{i}\right)$ data points [43-45] $\left(N_{A}\right.$ for each elemental group, at different $E_{i}$ energies), the same that are shown in Fig. 5. This corresponds to a number of degrees of freedom $\nu_{D}=75$, using the rigidity cut hypothesis (8).

In our fitting procedure we set $\lambda_{M}=1$ and we performed independent $\xi^{2}$ minimizations at different values of $\lambda_{D}$ within $\lambda_{D}=1$ and 0.01 , corresponding to different constraining power of the direct measurements to MACRO data. Under these conditions the minimization is successful and the calculation of the covariance matrix is accurate, at any $\lambda_{D}$ value, without parameter limits. The number of "equivalent data points" used in the multiparametric fit procedure is roughly given by $39+\lambda_{D} \cdot 91$. The contribution of the direct measurements to the fitting procedure, varying $\lambda_{D}$ from 1 to 0.01 , is reported in Table II. In this range of weight parameters, the $\xi^{2}$ function is equivalent to a $\chi^{2}$ variable with $\nu_{M}+\lambda_{D} \cdot \nu_{D}$ degrees of freedom (see Appendix B). 
TABLE II. Direct measurement contributions to the fitting procedure.

\begin{tabular}{cc}
\hline \hline$\lambda_{D}$ & Direct measurement contribution \\
\hline 1 & $91 / 130 \simeq 0.70$ \\
0.5 & $0.5 \cdot 91 / 85 \simeq 0.54$ \\
0.1 & $0.1 \cdot 91 / 48 \simeq 0.19$ \\
0.01 & $0.01 \cdot 91 / 40 \simeq 0.02$ \\
\hline \hline
\end{tabular}

Table III shows the main results of the multiparametric fit procedure. In this table the minimum of the $\xi^{2}$ function, the contributions to $\xi_{\min }^{2}$ from MACRO data $\left(\chi_{M}^{2}\right)$, the contribution from direct measurements $\left(\chi_{D}^{2}\right)$, the number of degrees of freedom, and the $\xi_{\text {min }}^{2}$ per degree of freedom at different $\lambda_{D}$ values are reported. This table shows that, according to this analysis, MACRO multimuon data and direct measurements are incompatible in the common range of primary energy. The contribution to $\xi_{\min }^{2}$ of the direct measurements ranges from $\chi_{D}^{2}=230$ at $\lambda_{D}=1$ to $\chi_{D}^{2}=260$ at $\lambda_{D}=0.01$. We show in Appendix $C$ that at $\lambda_{D} \sim 0.01$ the $\xi_{\min }^{2}$ roughly reaches its asymptotic limit $\left(\lambda_{D} \rightarrow 0\right)$ and therefore can be considered as determined by MACRO data alone.

We assume as the best fit of our data the primary cosmic ray composition model obtained from the multiparametric fit for $\lambda_{D}=0.01$ (hereafter referred to as the $A / R$ fit, $A / R$ standing for absolute muon rate), ${ }^{3}$ of which the basic parameters are summarized in Table IV. The full procedure to derive the flux parameters and their covariance matrix is detailed in Appendices A-D, which also include a complete list of the estimated errors and correlation coefficients in Tables VII and VIII, respectively. We note from Table VII that the spectra arising from the $A / R$ fit are actually due to multimuon data, since their parameters are in good agreement with the ones obtained with MACRO data alone (M/A fit).

\section{PRIMARY SPECTRA}

Figure 6 shows the elemental spectra arising from the $A / R$ fit, superimposed to the experimental data of the direct measurements used in the fit. In this figure the solid line gives the central value of the fit, the dashed lines represent the uncertainties on the spectra (one $\sigma$ errors) calculated using the covariance matrix of the parameters given by the fitting procedure. It can be seen that the spectra of the fit are consistent within errors with direct measurements for the three heaviest groups. For lighter elements the agreement with data points is achieved only at lower energies (below 10 $\mathrm{TeV}$ ), whereas the fitted spectra exceed direct data (mainly coming from a single experiment [43]), especially for $H$, at

\footnotetext{
${ }^{3} \mathrm{We}$ note that the composition models arising from the fitting procedure are similar in the whole range of $\lambda_{D}$. This fact demonstrates the dominant contribution of MACRO data in our constrained fit method, even for large values of $\lambda_{D}$. The main reason for this is that MACRO multimuon rates are determined by a convolution of the primary spectra of the different nuclei over a wide energy range (see Sec. II), while direct experiments measure single nuclei at fixed primary energy in a limited energy region.
}

TABLE III. Results of the fit at different $\lambda_{D}$ values $\left(\lambda_{M}=1\right)$.

\begin{tabular}{lccccc}
\hline \hline$\lambda_{D}$ & $\xi_{\min }^{2}$ & $\chi_{M}^{2}$ & $\chi_{D}^{2}$ & $\mathrm{DF}=\nu_{M}+\lambda_{D} \cdot \nu_{D}$ & $\xi_{\min }^{2} / N_{\mathrm{DF}}$ \\
\hline 1 & 243 & 13 & 230 & 98 & 2.48 \\
0.5 & 128 & 12.5 & 231 & 60.5 & 2.11 \\
0.1 & 35 & 10.9 & 241 & 30.5 & 1.15 \\
0.01 & 13.6 & 11 & 260 & 24 & 0.57 \\
\hline \hline
\end{tabular}

increasing energies. Therefore these groups are the main source of the incompatibility between the present analysis of multimuon data and direct measurements, which prevents us from fitting both data sets on the same ground (i.e., at $\lambda_{D}$ $=1$. We envisage two possible sources for this disagreement: (1) inadequate modeling of muon rates adopted in our simulation (this will be discussed in Sec. VII); (2) possible systematics in the direct measurements of light nuclei at the highest energies.

However, we want to stress that the individual spectra arising from our fit are correlated one with each other (see Table VIII) and then we believe that it is more sensible to compare our results with other experiments on the basis of "global" features (like the all-particle spectrum or the average mass composition) for which these correlations are less effective.

Figure 7 shows the all-particle spectrum arising from the $A / R$ fit, superimposed to previous experimental data: JACEE [43], Danilova [5], Grigorov [47], BASJE [8], Akeno [6], Tunka [9], MSU [7], Tibet AS $\gamma$ [10]. It can be easily recognized that the spectrum of the fitted model is higher and flatter than the one obtained from direct measurements alone (shown in Fig. 7 as a dashed area), exceeding it by an amount ranging from $15 \%$ at $E=10 \mathrm{TeV}$ to $50 \%$ at $E=100$ $\mathrm{TeV}$. The need of multimuon data for higher primary spectra already emerged in our previous analysis based on trial composition models [16]. A deficit of the order of at least $25 \%$ in the predicted rates has been observed therein using composition models tailored to render directly measured elemental abundances at $\leqslant 100 \mathrm{TeV}$. A remarkable feature of the reconstructed all-particle spectrum, which derives from our fitting procedure, is the consistency with EAS measurements around and above the knee. We emphasize that these measurements, shown in Fig. 7 for comparison, are not used in the minimized function. The fitted all-particle spectrum can be suitably represented with single power laws at primary

TABLE IV. Composition parameters obtained in the $A / R$ fit. The spectrum of each component is given by Eqs. (5) and (6) in the text. It has to be noted that only $E_{\text {cut }}(\mathrm{Fe})$ is actually fitted. The other energy cutoffs are reported for convenience, but are derived from Eq. (8).

\begin{tabular}{lcccc}
\hline \hline $\begin{array}{l}\text { Mass } \\
\text { group }\end{array}$ & $\begin{array}{c}K_{1} \\
\left(\mathrm{~m}^{-2} \mathrm{~s}^{-1} \mathrm{sr}^{-1} \mathrm{GeV}^{\gamma_{1}-1}\right)\end{array}$ & $\gamma_{1}$ & $\begin{array}{c}E_{\text {cut }} \\
(\mathrm{GeV})\end{array}$ & $\gamma_{2}$ \\
\hline $\mathrm{H}$ & $1.2 \times 10^{4}$ & 2.67 & $2.2 \times 10^{5}$ & 2.78 \\
$\mathrm{He}$ & $1.3 \times 10^{3}$ & 2.47 & $4.4 \times 10^{5}$ & 3.13 \\
$\mathrm{CNO}$ & $3.9 \times 10^{2}$ & 2.42 & $1.5 \times 10^{6}$ & 3.58 \\
$\mathrm{Mg}$ & $4.5 \times 10^{2}$ & 2.48 & $2.6 \times 10^{6}$ & 3.31 \\
$\mathrm{Fe}$ & $2.4 \times 10^{3}$ & 2.67 & $5.6 \times 10^{6}$ & 2.46 \\
\hline \hline
\end{tabular}



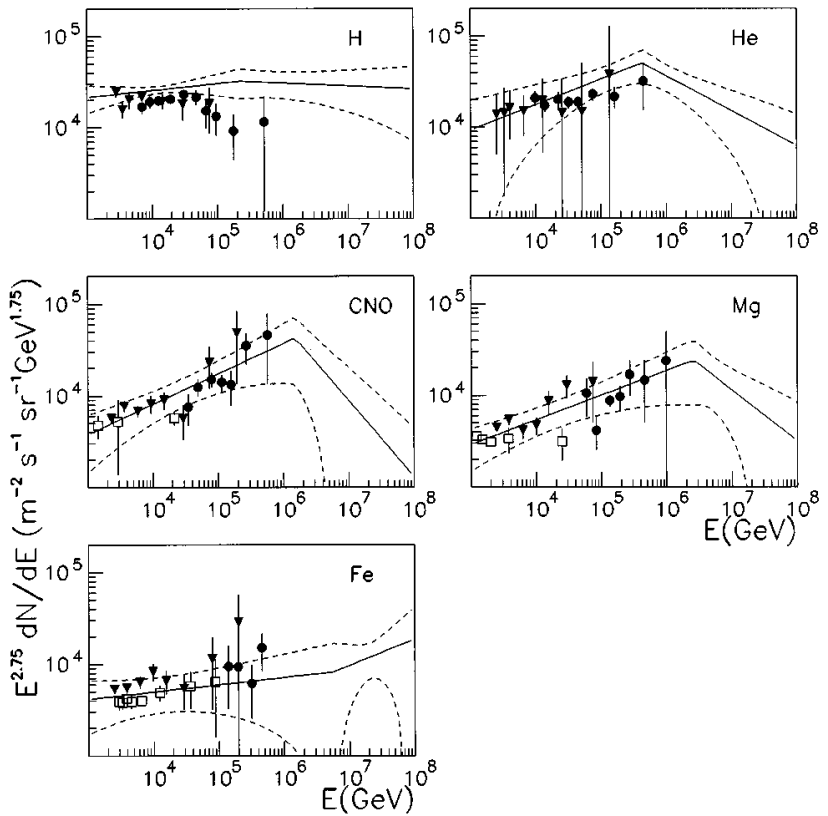

FIG. 6. Elemental spectra arising from the $A / R$ fit, superimposed to the experimental data of the direct measurements used in the fit. Solid lines: central value of the fit; dashed lines: spectra at $\pm 1 \sigma$ error, calculated using the covariance matrix of the parameters given by the fitting procedure. Symbols for direct measurements are the same as in Fig. 5.

energies far from the knee region, with a gradual change at intermediate energies. The corresponding spectral indexes are $2.56 \pm 0.05$ for $E<500 \mathrm{TeV}$ and $2.9 \pm 0.3$ for $E>5000$ $\mathrm{TeV}$.

We remind the reader that these results have to be considered valid in the context of our assumptions on Monte Carlo simulation. In Sec. VII we discuss how these assumptions, and in particular the hadronic interaction model, can affect our results.

\section{A. Sensitivity to the knee}

We verified that the rigidity cut hypothesis (8) assumed in the fitting procedure is consistent with our data by making a fit without this assumption. In this fit we fixed all the other parameters, but $E_{\text {cut }}(A)$, at the values shown in Table IV and we fitted independently $E_{\text {cut }}(A)$ releasing the relationship (8). The resulting values

$$
\begin{gathered}
E_{\text {cut }}(H)=10^{(5.3 \pm 0.6)} \mathrm{GeV}, \quad E_{\text {cut }}(\mathrm{He})=10^{(5.7 \pm 0.2)} \mathrm{GeV}, \\
E_{\text {cut }}(\mathrm{CNO})=10^{(6.2 \pm 0.1)} \mathrm{GeV}, \quad E_{\text {cut }}(\mathrm{Mg})=10^{(6.5 \pm 0.1)} \mathrm{GeV}, \\
E_{\text {cut }}(\mathrm{Fe})=10^{(6.8 \pm 0.5)} \mathrm{GeV}
\end{gathered}
$$

are compatible, within the errors, with the values reported in Table IV showing that MACRO data are consistent with the rigidity cut hypothesis.

As shown in Fig. 7 the all-particle spectrum arising from the MACRO composition analysis indicates the sensitivity of MACRO data to the knee. To prove this, we have performed a different fit under the assumption of a single power law for each group (corresponding to 10 free parameters). As a result we obtained a probability that $\xi^{2}$ exceeds $\xi_{\min }^{2}$ of $5.8 \%$ to be

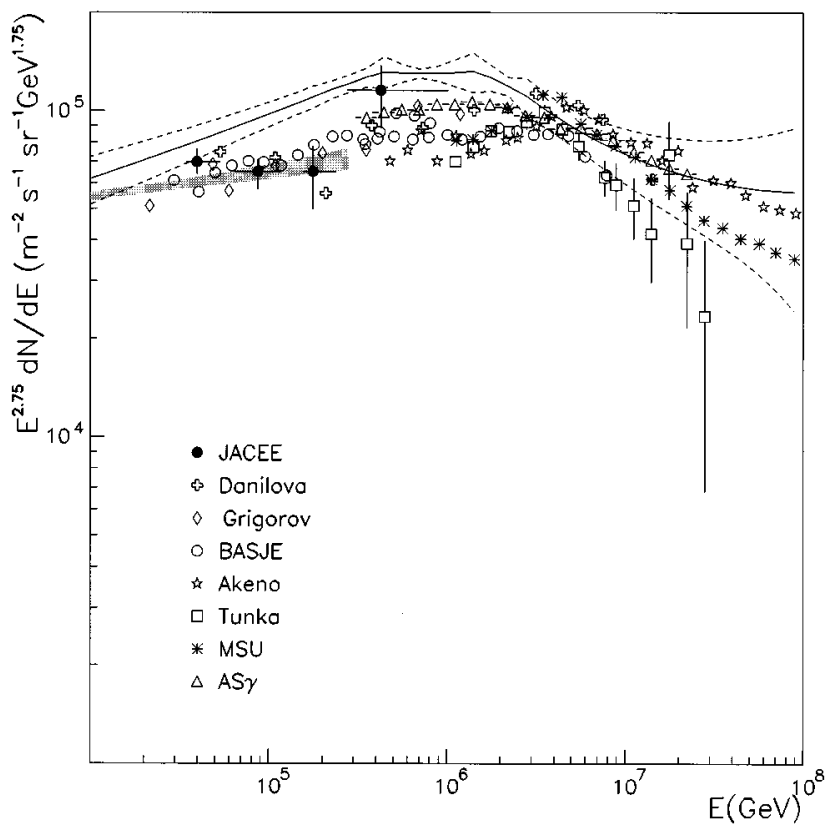

FIG. 7. All-particle spectrum arising from the $A / R$ fit (solid line: best value; dashed lines: $\pm 1 \sigma$ error) superimposed to previous experimental data: JACEE [43], Danilova [5], Grigorov [47], BASJE [8], Akeno [6], Tunka [9], MSU [7], Tibet AS $\gamma$ [10]. Dashed area: spectrum obtained from the fit of direct measurements shown in Fig. 5.

compared with the value of $95 \%$ in the case of the two spectral index hypothesis. This comparison implies that the change of the slope at the knee is preferred from MACRO data. It has to be emphasized that this result emerges directly from multimuon data, since, as reported in Sec. III B, single slope spectra give a good description of the data of direct measurements. This is a remarkable outcome of this analysis since for the first time an underground experiment shows sensitivity to the knee.

\section{B. Best fit procedure on the muon rate ratios}

In the fitting procedure described in the previous sections we used the absolute multiple muon rate measurements of the individual multiplicities. As reported in Secs. II B and II C the uncertainties on Monte Carlo predictions from the knowledge of the rock around MACRO and muon propagation through the rock affect the absolute muon rates, but they do not alter significantly the shape of the multiplicity distribution. Therefore a study in terms of the ratio between the rate at a certain multiplicity $R\left(N_{\mu}\right)$ and the rate of single muons $R(1)$ permits a cancellation of effects due to these systematic uncertainties. We therefore applied the multiparametric fit procedure to the muon rate ratios $r\left(N_{\mu}\right)$ $=R\left(N_{\mu}\right) / R(1)$, defining $\chi_{M}^{2}$, in Eq. (7), with $r\left(N_{\mu}\right)$ in place of $R\left(N_{\mu}\right)$. In this way only the shape of the multiplicity distribution is taken into account, while the absolute normalization of the primary fluxes is fixed by the data of the direct measurements. The all-particle spectrum arising from the fit of the rate ratios for $\lambda_{D}=0.01$ (hereafter referred to as the $R / R$ fit), is shown in Fig. 8. This spectrum has the same shape of the spectrum obtained from the fit of the absolute muon rates $(A / R$ fit), shown in Fig. 7 and, as expected, is in 


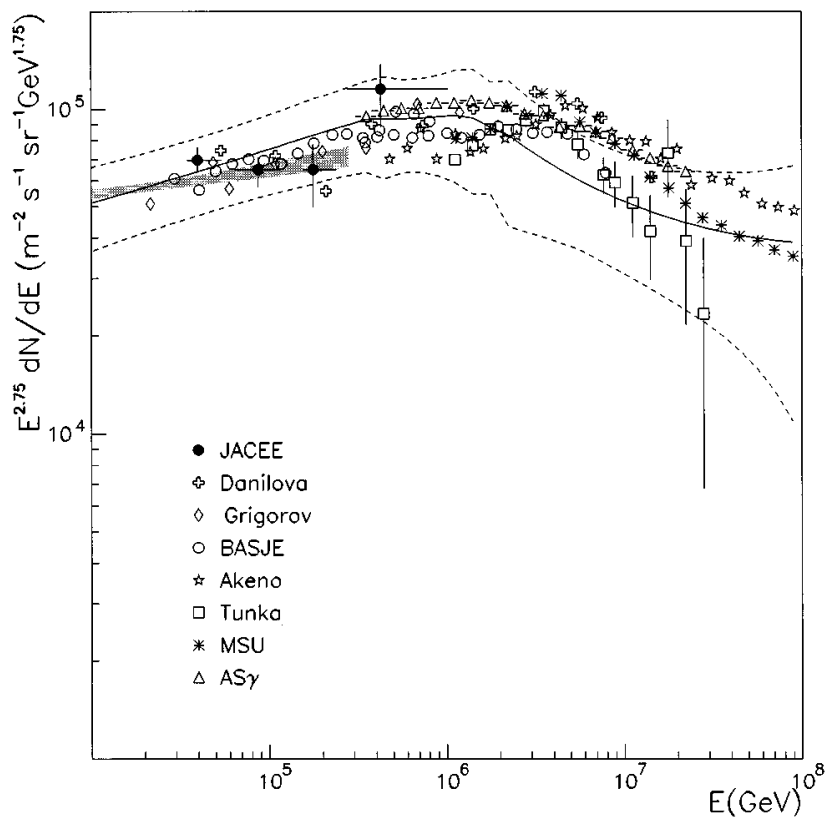

FIG. 8. All-particle spectrum arising from the $R / R$ fit, superimposed to previous experimental data. Symbols and line types are the same as in Fig. 7.

better agreement with the absolute normalization of the direct measurements. However, at higher energies, the spectrum shown in Fig. 8 is less consistent with the EAS measurements with respect to the one obtained from the fit of the absolute rates.

\section{PRIMARY COMPOSITION}

Figure 9 shows the dependence of the average mass number of cosmic rays $\langle A\rangle$ on primary energy. The dependence

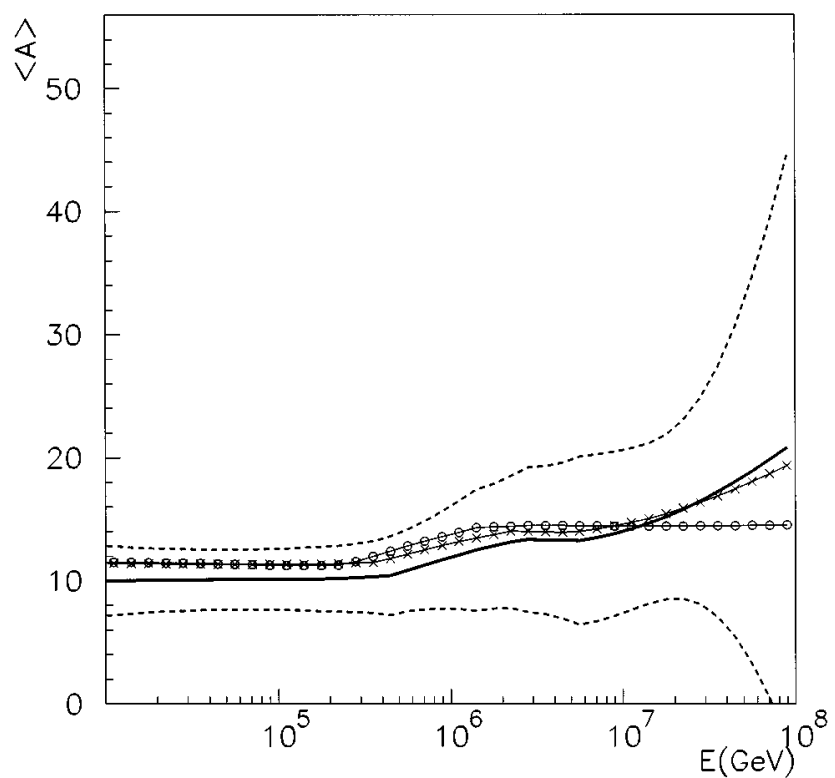

FIG. 9. Dependence of the average mass of cosmic rays on primary energy. Solid line: $\langle A\rangle$ arising from the fit of the absolute muon rates $(A / R$ fit $)$, dashed lines: $\langle A\rangle \pm 1 \sigma$ from the $A / R$ fit, line through crosses: $\langle A\rangle$ from the fit of the rate ratios $(R / R$ fit $)$, line through circles: $\langle A\rangle$ from the fit of MACRO data alone (M/A fit).
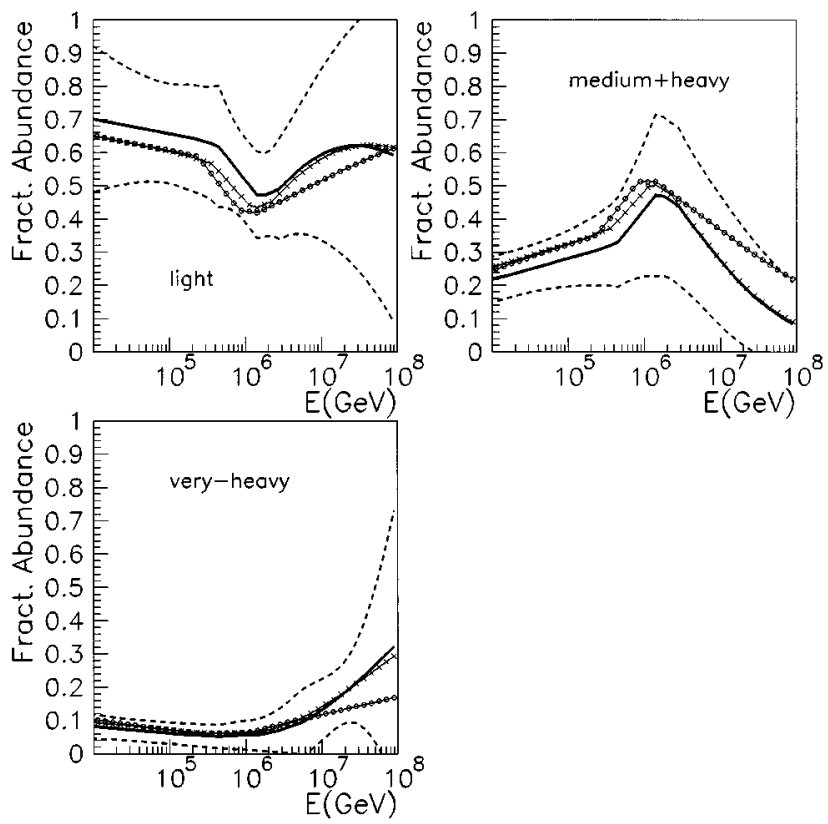

FIG. 10. Dependence on primary energy of the relative abundances of the light $(\mathrm{H}+\mathrm{He})$, medium-heavy $(\mathrm{CNO}+\mathrm{Mg})$ and veryheavy $(\mathrm{Fe})$ components. Line types are the same as in Fig. 9.

on primary energy of the relative abundances of the light $(\mathrm{H}+\mathrm{He})$, medium-heavy $(\mathrm{CNO}+\mathrm{Mg})$, and very-heavy $(\mathrm{Fe})$ components of the fitted models are given in Fig. 10. Both these figures refer to the fit of the absolute muon rates $(A / R$ fit): the solid line shows the best fit average mass and the dashed lines the same function at $\pm 1 \sigma$ error. For comparison the results obtained from the fit of the muon rate ratios $(R / R$ fit, line through crosses) and the fit of the MACRO data alone ( $M / A$ fit, line through circles) are also given. The results from the three fit procedures are very close to each other, in terms of $\langle A\rangle$ and relative abundances of mass groups. The closeness of the $A / R$-fit to the $M / A$-fit supports the convergence criterion used therein and confirms that the resulting composition is almost entirely determined by multimuon data. On the other hand, the comparison between the fits obtained using absolute and relative muon rates gives us confidence in the stability of our fitting procedure. Table $\mathrm{V}$ shows at different primary energies the relative abundances of each elemental group as estimated in the $A / R$ fit.

Both $\langle A\rangle$ and the relative abundances show little dependence, within errors, on the primary energy below $10^{6} \mathrm{GeV}$. At higher energies the best fit average mass shows a mild increase with energy, even though no definite conclusion can

TABLE V. Relative abundances of the different elemental groups in the $A / R$ fit.

\begin{tabular}{lccccc}
\hline $\begin{array}{l}E \\
(\mathrm{TeV})\end{array}$ & $\mathrm{H}$ & $\mathrm{He}$ & $\mathrm{CNO}$ & $\mathrm{Mg}$ & $\mathrm{Fe}$ \\
\hline 10 & $0.42 \pm 0.08$ & $0.28 \pm 0.19$ & $0.13 \pm 0.06$ & $0.09 \pm 0.03$ & $0.08 \pm 0.04$ \\
$10^{2}$ & $0.31 \pm 0.09$ & $0.34 \pm 0.11$ & $0.18 \pm 0.07$ & $0.11 \pm 0.04$ & $0.06 \pm 0.03$ \\
$10^{3}$ & $0.23 \pm 0.08$ & $0.29 \pm 0.09$ & $0.29 \pm 0.17$ & $0.14 \pm 0.08$ & $0.05 \pm 0.04$ \\
$10^{4}$ & $0.35 \pm 0.17$ & $0.21 \pm 0.14$ & $0.16 \pm 0.15$ & $0.16 \pm 0.11$ & $0.12 \pm 0.08$ \\
\hline
\end{tabular}




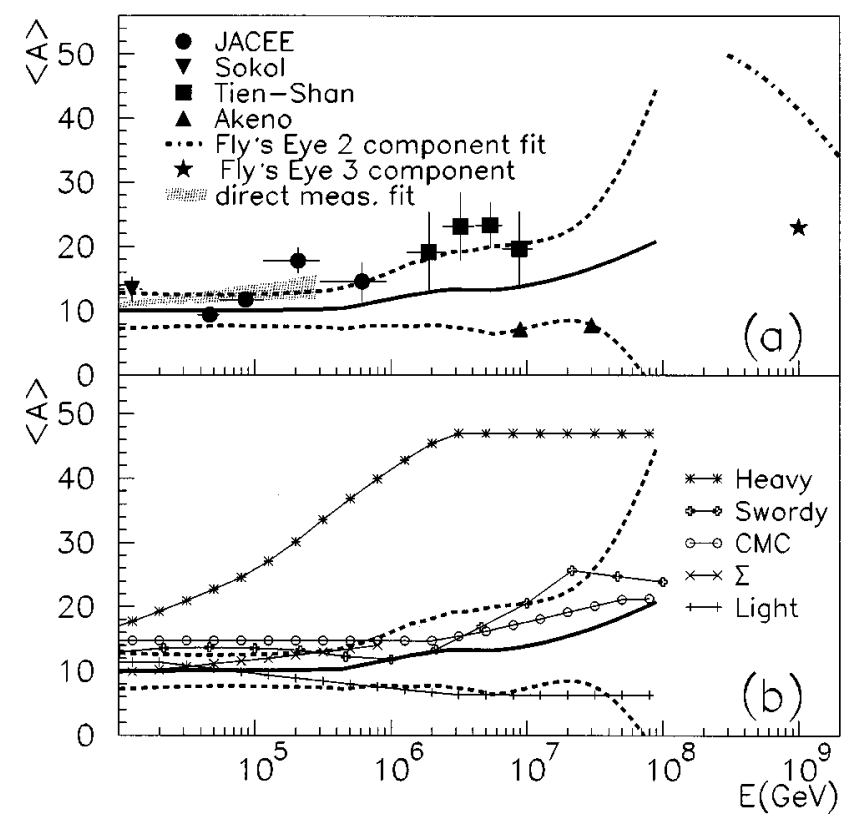

FIG. 11. Comparison of the average primary mass arising from our $A / R$ fit (solid line: central value; dashed line: value at one sigma error) with other measurements (a) and predictions (b). The $\Sigma$ model is limited to primary energies below the knee, being obtained from a fit of direct measurements. References are given in the text.

be reached taking into account the increasingly large uncertainties from the fit.

In Fig. 11, $\langle A\rangle$ obtained in our $A / R$ fit is compared with other measurements and predictions. In particular, Fig. 11(a) shows that our $\langle A\rangle$ estimate is consistent, within errors, with the ones obtained from the fit of direct measurements (shown as dashed area in the figure) and from other composition analyses of direct observations $[43,44]$ and EAS array data $[48,49]$, in the same primary energy region. In this figure $\langle A\rangle$ is displayed up to $\sim 10^{9} \mathrm{GeV}$, exceeding the region covered by MACRO by more than one decade, in order to include the composition results from Fly's Eye [50] in the $\mathrm{EeV}$ region. The dash-dotted line gives the $\langle A\rangle$ dependence on primary energy arising from a two-component fit of the ratio iron flux/proton flux as obtained from Fly's Eye elongation data between $10^{17.5}$ and $10^{19.5} \mathrm{eV}$. A three-component analysis, with the insertion of a $\mathrm{CNO}$ component and fixing the $\mathrm{Fe}$ contribution to $40 \%$, gives the point represented with a star.

In Fig. 11(b) the fitted $\langle A\rangle$ is compared with different composition models: the light and heavy models [18], already used as extreme compositions $[16,15,17]$; the constant mass composition (CMC) model [19]; the $\Sigma$ model, which is the composition preferred in the EASTOP/MACRO coincidence analysis [20]; a recent model proposed by Swordy [51] in the context of a simple leaky box model, incorporating a cutoff propagation pathlength to account for the unobserved anisotropy at high energies. This comparison confirms that MACRO data exclude a primary composition that becomes heavier with increasing energy, as dramatically as does the heavy model. Nevertheless the achieved resolution prevents us from discriminating among different models that, though originating from different physical hypotheses, are character-

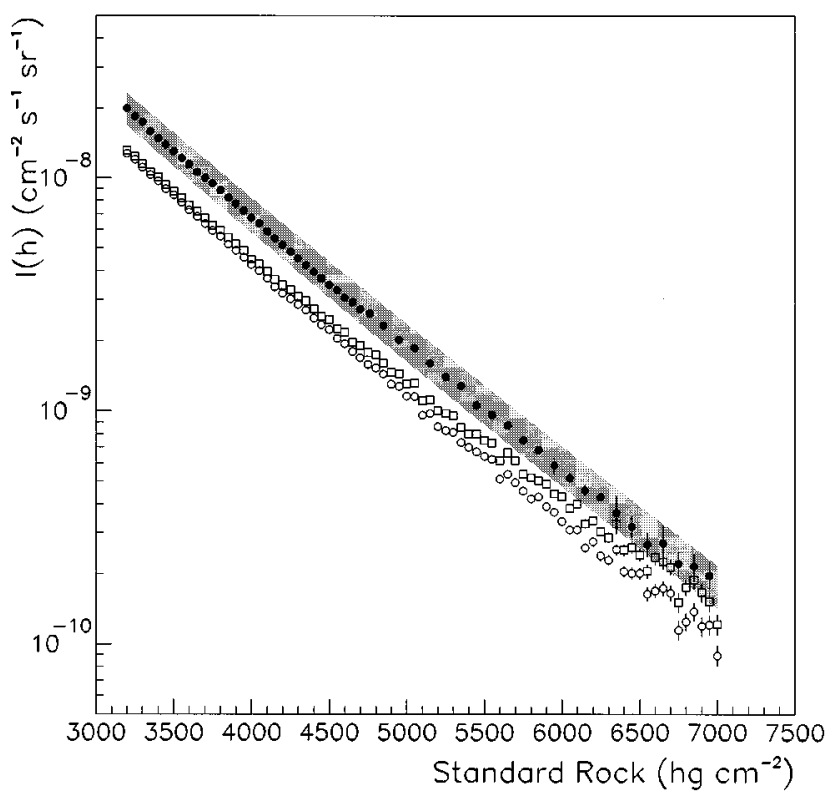

FIG. 12. MACRO vertical depth intensity data points, compared with the results of Monte Carlo simulations. Full circles: experimental data; open squares: light model; open circles: heavy model; dashed area: $A / R$ fit model within $\pm 1 \sigma$ errors on the fitted parameters.

ized by a flat or slowly increasing $\langle A\rangle$ as a function of the primary energy.

\section{COMPOSITION DEPENDENCE OF OTHER MUON FEATURES}

For indirect measurements of primary cosmic ray composition, it is important to identify as many experimental observables as possible which can help in the validation of the composition models. In order to test the fitted composition model (the $A / R$ fit model), our experimental results on vertical muon intensity and decoherence function have been compared with the predictions of this model.

\section{A. The depth intensity curve}

The depth intensity curve can contribute to the knowledge of the primary cosmic ray composition and can furnish further information on the absolute normalization. As discussed in [37,21], the MACRO experimental vertical muon intensity as a function of depth agrees with the world average within a few percent, so that the comparison between our data and the results of a Monte Carlo simulation confirms our understanding of detector acceptance and overburden systematics. Figure 12 shows that two extreme models, the heavy and light [18] ones, which are based on direct measurements as starting points for extrapolation to higher energies, are inconsistent with the experimental behavior of the depth intensity curve. Assuming the MACRO composition model derived from the $A / R$ fit, we obtain good agreement between the predictions of the Monte Carlo simulation and the experimental data, both in absolute normalization and slope. This scenario is a further confirmation of the results of our previous analyses based on trial models $[16,15,17]$. 


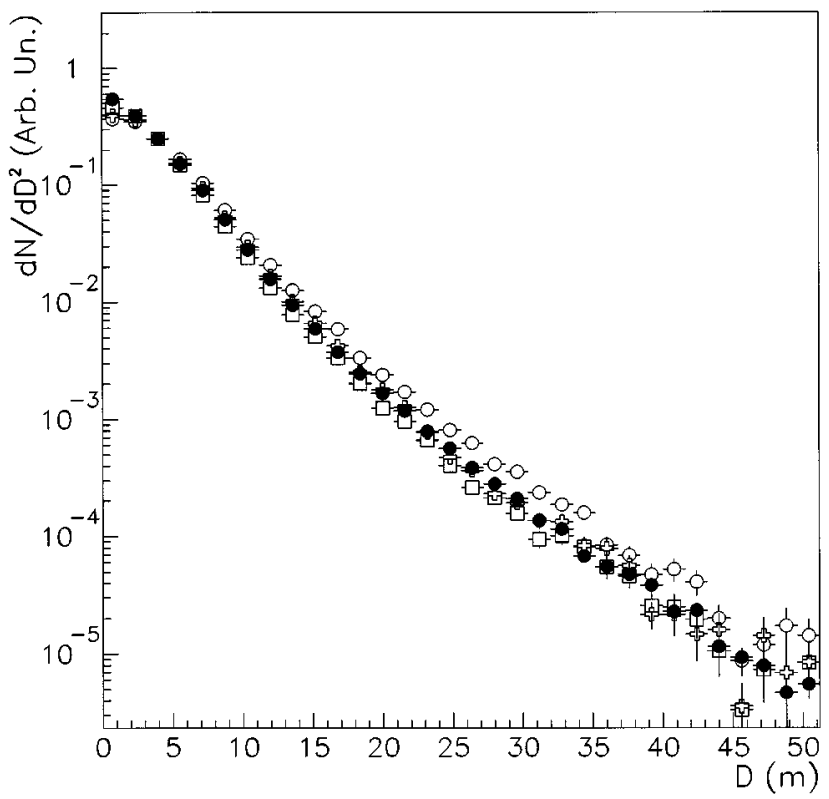

FIG. 13. Comparison between experimental and predicted muon decoherence distributions $d N / d D^{2}$ measured in MACRO ( $D$ being the muon pair separation), for events with any multiplicity. Full circles: experimental data; open squares: light model; open circles: heavy model; open crosses: $A / R$ fit model. Here each muon pair enters with a $1 / N_{\text {pair }}$ weight, where $N_{\text {pair }}$ is the number of unambiguously reconstructed pairs. Experimental and predicted distributions, normalized one to each other, are given in arbitrary units.

\section{B. The decoherence curve}

The shape of the separation distribution of muon pairs measured by MACRO has been demonstrated to be sensitive to hadronic interaction models, and has allowed a validation of the HEMAS interaction model [25,26]. A limited dependence on the composition model is also shown in these references. In Fig. 13 we plot the measured decoherence distribution compared to the predictions from different composition models. Here each multiple muon event having $N_{\text {pair }}$ unambiguously reconstructed pairs has unit weight, i.e., each pair enters with a weight $1 / N_{\text {pair }}$ [21] and the different distributions are normalized one to the other in order to emphasize the comparison of shape. Simulated events have been generated, using the HEMAS hadronic interaction model, according to different composition models: the light and heavy models [18] and the model arising from our $A / R$ fit. We notice that the $A / R$ fit model also provides the best agreement to the shape of the decoherence distribution in space. This is significant, since we do not use the muon separation data in the fit.

However, we note that the normalization of the decoherence curve is also of interest, since it introduces additional sensitivity to the spectrum and composition of primary cosmic rays. The primary composition has different effects on the muon flux and decoherence curve normalization. The inclusive muon flux tends to be dominated by the proton part of the primary composition, while other nuclei, if present, give large contributions to the decoherence curve [28]. This is due to the fact that in the decoherence curve a single event of high multiplicity multiplicity $N_{\mu}$ weights heavily by producing $N_{\mu}\left(N_{\mu}-1\right) / 2$ pairs. Therefore the decoherence function receives a large contribution from the richest bundles

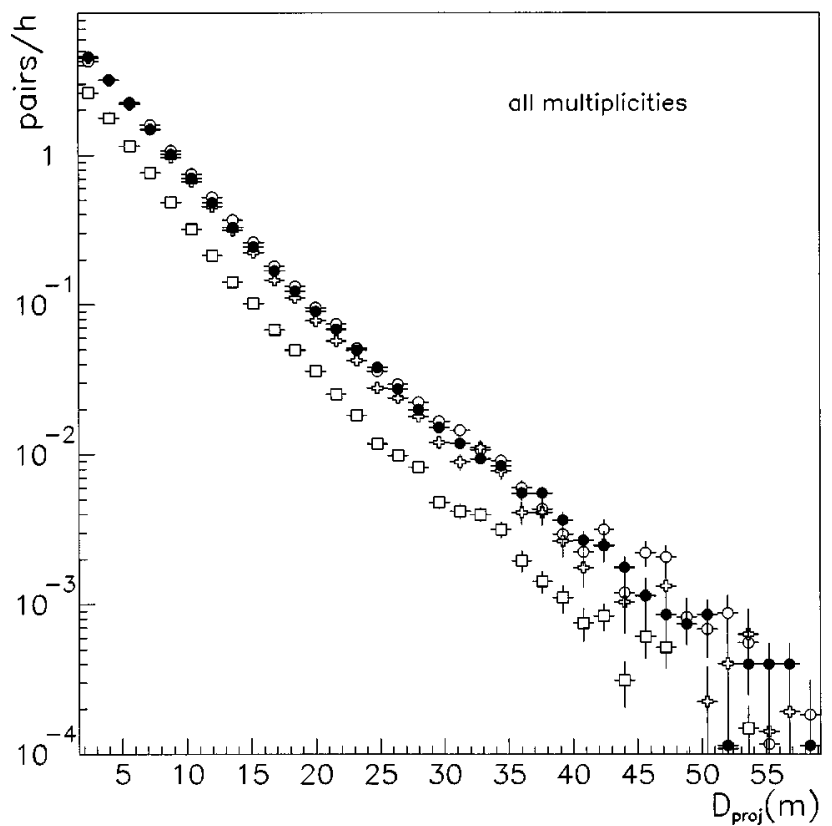

FIG. 14. Comparison between experimental and predicted muon lateral separations, in MACRO, projected in the wire view for events with any multiplicity. Same symbols as in Fig. 13. Projected separations lower than $1 \mathrm{~m}$ have not been considered because of experimental inefficiencies in the close track separation. Here each muon pair enters with unit weight.

and then is dominated by the contribution of the heavy primary nuclei, reflecting the highest primary energy region to which MACRO is sensitive.

As discussed in the companion paper [21], decoherence analysis at high multiplicity can be performed without bias by MACRO only by considering the muon separation inside the detector, projected in one view, in order to override problems with unequal efficiencies of reconstructing unambiguously muon pairs at different multiplicities. In Fig. 14 the MACRO experimental muon lateral separation projected in the wire view is shown superimposed to Monte Carlo predictions, for events with any multiplicity. The different normalization of the distributions obtained with the two "extreme" models, in conjunction with the shape analysis described above, show sensitivity of the muon lateral distribution to primary composition. The prediction of the light model is in disagreement with the experimental data. The normalizations of the heavy and the $A / R$ fit models are in good agreement with the experimental data, apart from the shape consideration derived from Fig. 13.

Enhanced sensitivity to primary composition is achieved from the study of the same distribution as a function of the multiplicity. In Fig. 15 the comparison between experimental and Monte Carlo distributions for events with multiplicities $N_{\mu}=2, N_{\mu}=3, N_{\mu}=4-6$, and $N_{\mu}=7-20$ is shown. From these comparisons one can extract interesting information. For low multiplicity events the predictions of the two "extreme" models are very similar, while the sensitivity to primary composition increases at higher multiplicities. The light model, which contains a very small fraction of heavy nuclei, underestimates the rate of muon pairs in the whole separation range, independent of the event multiplicity. On the contrary the heavy model, which contains a large fraction 


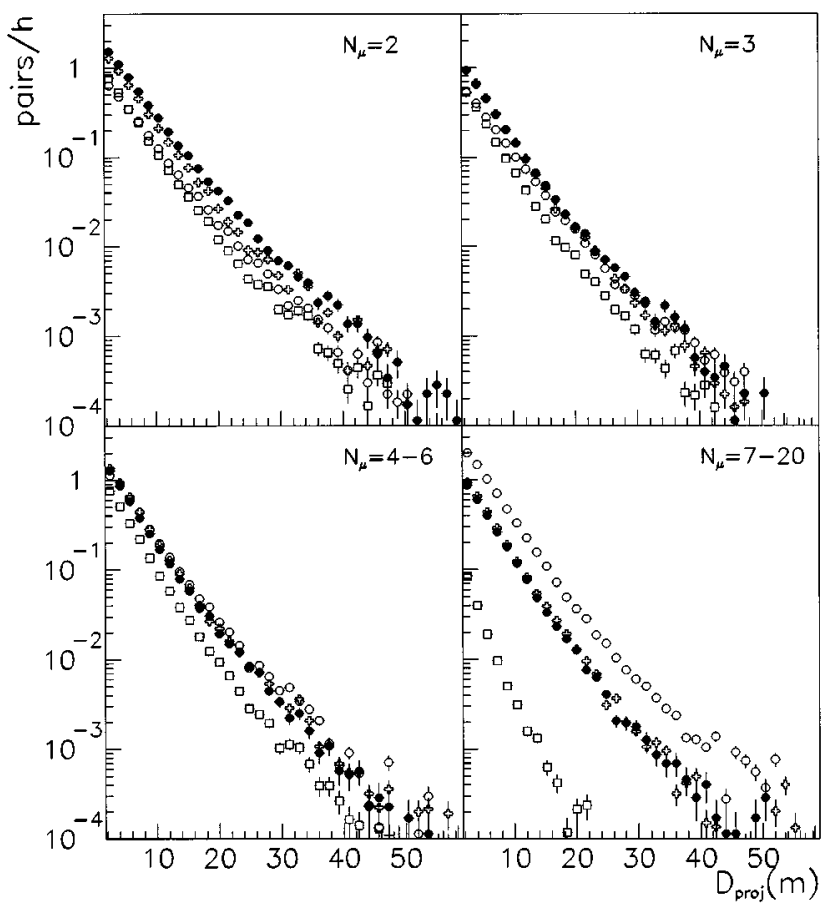

FIG. 15. Comparison between the experimental muon lateral separations, in MACRO, projected in the wire view as a function of the event multiplicity. Same symbols as in Fig. 13. Here each muon pair enters with unit weight.

of heavy nuclei, has a different behavior. The predictions of this model are very similar to those of the light model for $N_{\mu}=2$. For $N_{\mu}=3$ it predicts a rate of muon pairs lower than the experimental data for separations $\leqslant 10 \mathrm{~m}$, but is in good agreement with experimental data for larger separations. For multiplicities $N_{\mu}=4-6$ the heavy model agrees with experimental data in the whole separation range. Increasing the multiplicity $\left(N_{\mu}=7-20\right)$, the heavy model overestimates the experimental rates, clearly indicating that this model contains an excess of heavy nuclei with respect to the real content of these nuclei in the primary cosmic rays. We also notice that at high multiplicity the shapes predicted by the light and heavy models are different from the measured ones. The MACRO $A / R$ fit model reproduces the lateral distribution of experimental data in the whole multiplicity range both in shape and normalization. This is an important result of this analysis, since it shows that the composition estimated from muon rates only is consistent also with higher order features of multimuon events.

\section{DISCUSSION}

We have pointed out that our results exhibit some disagreement with the existing direct measurements in the primary energy region $10-100 \mathrm{TeV}$. We cannot discuss results from other experiments here, but we show outcomes resulting from a deep investigation about the uncertainties which can affect the calculation of our multimuon rates. As already mentioned in Sec. II, these are uncertainties in the hadronic interaction model, in the knowledge of the map of the rock around MACRO, and in muon propagation through the rock. The uncertainties from the knowledge of the rock around MACRO and the muon propagation through the rock can be canceled by applying the best fit procedure to the muon rate ratios. In Secs. IV B and V we have shown that the best fit of these ratios does not alter the main conclusions reached with the absolute rates (e.g., spectral indexes and average primary mass dependence on energy). Therefore the main contribution to systematic uncertainties in our Monte Carlo simulation is due to the hadronic interaction model that could eventually act differently in the various energy regions. In Sec. II A this effect is estimated to be of the order of about $10 \%$ in the predicted multimuon rates. In order to study the dependence of our results from the best fit procedure on the adopted hadronic interaction model, we applied the multiparametric fit procedure using the SIBYLL code as hadronic interaction model. The predicted multimuon rates (3) have been calculated using probabilities $D_{A}\left(E, N_{\mu}\right)$ calculated with SIBYLL on a reduced sample of simulated events, in place of those calculated with HEMAS. The all-particle spectrum arising from the fitting procedure assuming the SIBYLL model is at most of the order of $10 \%$ lower than the one shown in Fig. 7, obtained with HEMAS. This value can be considered as an estimate of the systematic uncertainty in our fitting procedure. Nevertheless the results from the fit using the SIBYLL model are very similar to the corresponding ones with HEMAS, in terms of $\langle A\rangle$ and relative abundances of mass groups.

Important sources of uncertainties in the hadronic interaction model (HEMAS, SIBYLL, and in general any other model) come from our limited knowledge of proton air and nucleusair inclusive meson production at very high energies. A model for hadronic and nuclear interactions to be used in cosmic ray physics should work from the pion production threshold up to the highest possible primary energies. Experimental results from collider and fixed target experiments at accelerators provide important inputs up to a proton energy $E_{p} \sim 1000 \mathrm{TeV}$. However, in the highest energy part of the energy region investigated in this search, which corresponds to center-of-mass energies $\sqrt{s} \approx 10 \mathrm{TeV}$ [i.e., energies reached at the CERN Large Hadron Collider (LHC) or greater] no direct collider measurements are yet available, and lower energy data must be extrapolated. Nucleusnucleus data from accelerator experiments need a much stronger extrapolation. This situation could lead to the belief that possible inadequacies of interaction models, that are tailored to experimental data, are increasing with energy, but are virtually absent in the energy region below the knee. Indeed our results obtained from the $A / R$ fit show the strongest discrepancies with existing cosmic ray data just in that region.

A more careful study about hadronic interaction mechanisms shows that possible uncertainties are also present at lower energies. For the relevant kinematical region accessed (e.g., the Feynman- $x$ interval), there are important differences between cosmic ray cascades and particle production at accelerators. At colliders, the central region in hadronhadron collisions is usually best measured. At lower energies, in fixed target experiments, the forward fragmentation region is more easily accessible, yet very little data are available at $x_{F}$ exceeding 0.1. Multiple muons observed in underground detectors come from different kinematical regions determined by the energy of primaries that produce the muons. In particular, one can see that multimuon events 
TABLE VI. Average $x_{F}$ of the parent of the muons at MACRO depth, in different ranges of primary energy. Events generated using the HEMAS interaction model.

\begin{tabular}{lccc}
\hline \hline$E(\mathrm{TeV})$ & $N_{\mu}=1$ & $N_{\mu}=2-4$ & $N_{\mu} \geqslant 5$ \\
\hline$<10$ & 0.41 & 0.34 & \\
$10-10^{2}$ & 0.25 & 0.19 & 0.09 \\
$10^{2}-10^{3}$ & 0.23 & 0.19 & 0.16 \\
$10^{3}-10^{4}$ & 0.22 & 0.20 & 0.18 \\
$10^{4}-10^{5}$ & 0.20 & 0.20 & 0.19 \\
\hline \hline
\end{tabular}

originating from less energetic primaries are preferentially produced from parents in the very forward fragmentation region, whereas at higher primary energies the corresponding production kinematical region is at lower $x_{F}$. Table VI shows this fact at different primary energies and for a few groups of muon multiplicities. In particular it can be recognized that the highest $x_{F}$ parents are the main contributors of the low multiplicity muon events and then largely determine the inclusive muon rates. Therefore possible inadequacies of the hadronic interaction models in the far fragmentation region could, at least partly, explain the discrepancies between multimuon results and direct measurements.

\section{CONCLUDING REMARKS}

The multiparametric fit of muon rates is a successful tool for studying the primary cosmic ray composition. Having chosen a priori the HEMAS interaction model, our results are the following.

Multimuon data require the contribution of all the elemental groups in order to explain the observed muon rates in a wide multiplicity range. An important outcome of our fitting procedure is that MACRO data show sensitivity to the energy steepening above the knee.

The fitted all-particle spectrum exceeds, in the lower energy region, direct measurements by $\sim 10 \%$ at $10 \mathrm{TeV}$ to $\sim 50 \%$ at $100 \mathrm{TeV}$. Above $\sim 5000 \mathrm{TeV}$ it shows a good consistency with EAS array measurements. The spectral indexes of the fitted energy spectrum are $2.56 \pm 0.05$ for $E<500 \mathrm{TeV}$ and $2.9 \pm 0.3$ for $E>5000 \mathrm{TeV}$ with a gradual change at intermediate energies. The same fit procedure, applied to the ratios of muon rates to single muons $\left[R\left(N_{\mu}\right) / R(1)\right]$, permits cancellation of systematic effects related to muon propagation through the rock. In this case the fitted spectrum shows a better agreement with low energy measurements, but the spectral indexes are roughly the same as in the fit of the absolute rates.

Both $\langle A\rangle$ and the relative abundances show little dependence on the primary energy below about $1000 \mathrm{TeV}$. At higher energies the best fit average mass shows a mild increase with energy, even though no definite conclusion can be reached taking into account the increasingly large uncertainties deriving from the fit.

We have shown that the most important source of uncertainties comes from the choice of the hadronic interaction model. A comparison with a more recent model, SIBYLL, based on different physical assumptions, leads to spectra differing of the order of $10 \%$, but with a very similar composition. These differences in the $\mathrm{TeV}$ muon yields are consistent with what has been previously discussed by other authors. It is therefore likely that the disagreement between our underground muon analysis and direct measurements cannot be solved in a straightforward way. In our opinion this fact offers interesting hints which motivate further phenomenological and experimental studies.

\section{APPENDIX A: $\xi^{2}$ MINIMIZATION}

The estimate of the elemental flux parameters has been performed through a minimization of the function $\xi^{2}$ [see Eq. (9)], in which these parameters appear in two distinct terms: (1) a $\chi^{2}$ term $\left(\chi_{M}^{2}\right)$ based upon muon multiplicity rates from MACRO data (7). This term includes the flux parameters in the convolution integral (3); (2) a $\chi^{2}$ term $\left(\chi_{D}^{2}\right)$ built up with direct measurements of individual spectra (10).

The two pieces are used to define the minimized $\xi^{2}$ function through a linear combination, with fixed (i.e., not estimated) weight parameters. However the two contributions have quite a different role in our best fit procedure. The first is the primary term from which we want to estimate our parameters and then the corresponding weight $\left(\lambda_{M}=1\right)$ is not changed during the fit process. The second term has the role of constraining the flux parameters to a limited region of the parameter space and therefore the corresponding weight $\left(\lambda_{D}\right)$ is changed. It is straightforward that setting $\lambda_{D}$ to 0 is equivalent to a best fit of MACRO data alone.

The adopted procedure of constraining the flux parameters with external measurements has been suggested by the following considerations.

(i) The high number of free parameters and the dependence of the muon rates on the composition parameters, through a convolution of primary spectra over a wide energy range, prevent us from estimating the primary composition from the minimization of the $\chi_{M}^{2}$ function with free parameters.

(ii) The application of limits on parameters could in principle solve the previous problem and then prevent the parameters from taking on unphysical values. The minimization with limited parameters is generally (e.g., in MINUIT [41]) achieved with a nonlinear transformation of the parameters themselves and unavoidably introduces additional numerical difficulties to the process. Then the success of such minimization strongly depends on the minimized function and, in any case, it is recommended to release the boundary conditions after the minimum is reached, in order to get a reliable calculation of the covariance matrix. In our case this procedure turned out to be unsuccessful, mainly because of numerical problems arising from the intrinsically strong correlations among the parameters in the two-power-law function. Furthermore, the functional form of elemental spectra prevents an appropriate definition of the parameters boundaries. As a result, minimizations performed using MACRO data alone $\left(\xi^{2}=\chi_{M}^{2}\right)$ with limited parameters gave us spectra in reasonable agreement with the ones obtained with the constrained fit, but with parameter errors unreliable on account of unsolved numerical inaccuracies (e.g., covariance matrix not positive definite).

(iii) The minimization process at decreasing values of $\lambda_{D}$ showed a limiting minimum of $\xi^{2}$ at a value of $\lambda_{D}$ for which the minimization is still good and the evaluation of the covariance matrix is accurate (see Appendix C). 
Particular care has been taken in order to render the calculation of the $\xi^{2}$ function as free as possible of numerical inaccuracies. In our analysis, depending on the actual representation of the elemental fluxes (5) and (6), the $\xi^{2}$ calculation can be affected by rounding off errors due to the finite word length in the computer analysis. In particular, the result of the minimization process strongly depends on the energy scale which is used therein. The standard scale that is used in UHE C.R. literature, with primary energy in $\mathrm{GeV}$, is inappropriate for our calculation. In fact, our multimuon rates are sensitive to primary energies above a few $10 \mathrm{TeV}$ and the expected spectra rapidly fall off with energy. An energy scale of $10 \mathrm{TeV}$ or more is also to be preferred in order to avoid powers of too high energy values. We found that a scale between a few $\mathrm{TeV}$ and a few ten $\mathrm{TeV}$ is preferred in our fit; our final choice was an energy unit of $10 \mathrm{TeV}$. We also redefined the flux parameters in order to render them all of the same size. The spectral indexes $\left[\gamma_{1}(A)\right.$ and $\left.\gamma_{2}(A)\right]$ are expected to be of the order of few units and therefore we applied modifications to the other parameters to have them in the same range. This choice produces a covariance matrix calculated with good numerical accuracy. The spectra used in our minimization have the form

$$
\begin{aligned}
& \Phi_{A}(E)=10^{-3} \kappa_{1}(A)(E / 10 \mathrm{TeV})^{-\gamma_{1}(A)} \\
& \text { for } E<10^{\Lambda_{\text {cut }}(A)+1} \mathrm{TeV}, \\
& \Phi_{A}(E)=K_{2}(A)(E / 10 \mathrm{TeV})^{-\gamma_{2}(A)} \\
& \text { for } E>10^{\Lambda_{\text {cut }}(A)+1} \mathrm{TeV} .
\end{aligned}
$$

$\kappa_{1}(A), \gamma_{1}(A), \gamma_{2}(A)$, and $\Lambda_{\text {cut }}(A)$ are the parameters that are actually estimated. The new parameters are related to the ones in Eqs. (5) and (6) as

$$
\begin{gathered}
K_{1}(A)=10^{-3} \kappa_{1}(A)(10 \mathrm{TeV})^{\gamma_{1}(A)}, \\
E_{\text {cut }}(A)=10^{\Lambda_{\text {cut }}(A)}(10 \mathrm{TeV}) .
\end{gathered}
$$

\section{APPENDIX B: $\xi^{2}$ FUNCTION}

The $\xi^{2}$ function defined in Eq. (9) is not properly a $\chi^{2}$ variable as it results from a linear combination of two distinct $\chi^{2}$ components. We calculated the probability distribution of such a variable as

$$
p\left(\xi^{2}\right)=\int_{0}^{\xi^{2} / \lambda_{M}} \mathcal{P}_{\nu_{M}}\left(\chi_{M}^{2}\right) \frac{1}{\lambda_{D}} \mathcal{P}_{\nu_{D}}\left(\frac{\xi^{2}-\lambda_{M} \chi_{M}^{2}}{\lambda_{D}}\right) d \chi_{M}^{2}
$$

where $\mathcal{P}_{\nu}\left(\chi^{2}\right)$ is the probability of $\chi^{2}$ with $\nu$ degrees of freedom. In Fig. 16 we show the cumulative probabilities

$$
P\left(\geqslant \xi^{2}\right)=\int_{\xi^{2}}^{\infty} p(z) d z
$$

as obtained from Eq. (B1), in the range of $\lambda_{M}$ and $\lambda_{D}$ we actually used in our fit $\left(\lambda_{M}=1\right.$ and $\left.\lambda_{D}=1-0.01\right)$. It can be easily recognized that, in this range, our cumulative prob-

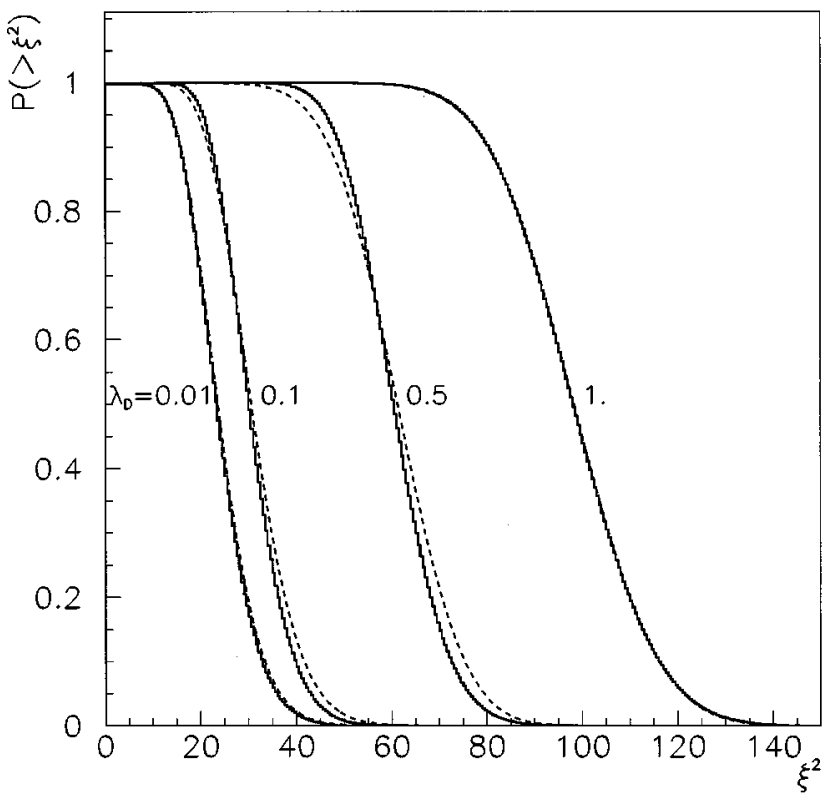

FIG. 16. Cumulative probability distributions $P\left(\geqslant \xi^{2}\right)$ [Eq. (B2), in the text] for different values of $\lambda_{D}$ (solid histograms). For comparison, dashed lines show the cumulative $\chi^{2}$ probabilities for $\lambda_{M} \nu_{M}+\lambda_{D} \nu_{D}$ degrees of freedom.

abilities are very close to, and in some cases indistinguishable from, the cumulative $\chi^{2}$ probability with $\lambda_{M} \nu_{M}$ $+\lambda_{D} \nu_{D}$ degrees of freedom (dashed curves):

$$
P\left(\geqslant \xi^{2}\right) \approx \int_{\xi^{2}}^{\infty} \mathcal{P}_{\lambda_{M} \nu_{M}+\lambda_{D} \nu_{D}}\left(\chi^{2}\right) d \chi^{2} .
$$

This means that, in the range of weight parameters considered in our fit, $\xi^{2}$ behaves as a $\chi^{2}$ variable with a number of degrees of freedom (DF) given by

$$
\nu=\lambda_{M} \nu_{M}+\lambda_{D} \nu_{D}
$$

\section{APPENDIX C: $\xi_{\min }^{2}$ AS A FUNCTION OF $\lambda_{D}$}

In Appendix A we described the procedure we used to estimate the flux parameters through a constrained fit of MACRO multimuon data. For each value of $\lambda_{D}(\leqslant 1)$ a minimum of the $\xi^{2}$ function has been calculated using the MINUIT minimization package. The minimization turns out to be successful up to very low values of $\lambda_{D}$, but numerical problems, mainly in the calculation of the covariance matrix, prevent us from obtaining reliable parameters and errors for $\lambda_{D}<0.01$. Figure 17 shows the values of $\xi_{\min }^{2}$ (full circles) reached at different values of $\lambda_{D}$. A solid line shows the corresponding number of degrees of freedom calculated according to Eq. (B4).

It is evident from this figure that $\xi_{\min }^{2}$ has an asymptotic behavior with $\lambda_{D}$ and reaches a minimum value already at $\lambda_{D} \sim 0.01$. At this value the minimum can be considered a reasonable estimate of the limiting value for $\lambda_{D} \rightarrow 0$ and then to be approximately free of the constraints from the 


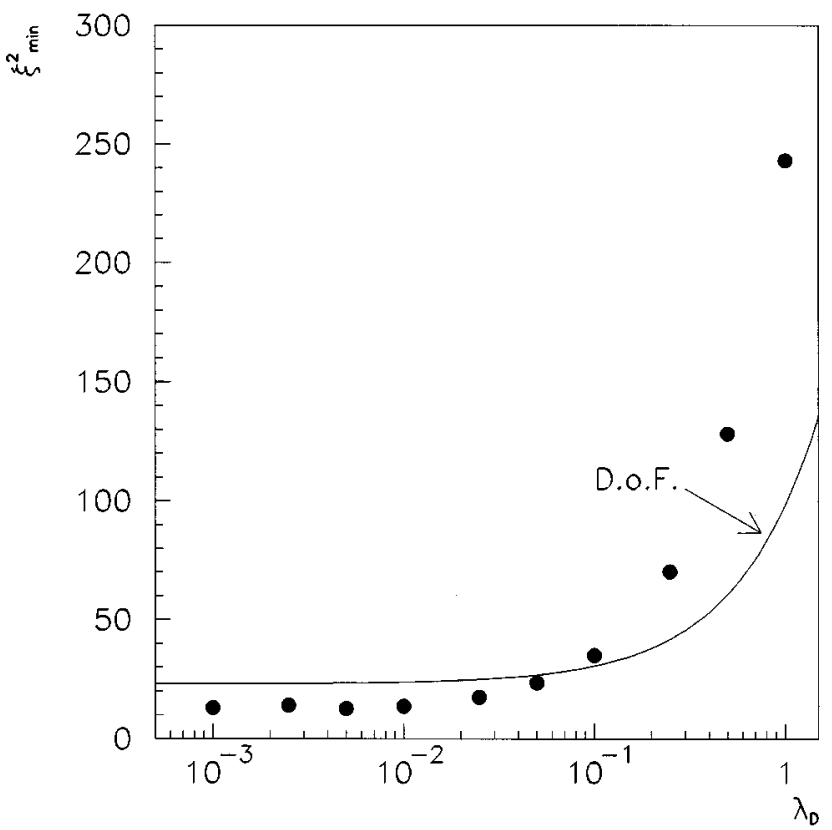

FIG. 17. $\xi_{\min }^{2}$ (full circles) as a function of the weight parameter $\lambda_{D}$. The solid curve shows the number of degrees of freedom calculated according to Eq. (B4).

$\chi_{D}^{2}$ component. This occurrence is of remarkable importance for our purposes since it allows us to obtain a reliable estimate of the parameter errors otherwise affected by unsolvable numerical problems. On the other hand, the best fit parameters show a very weak dependence on $\lambda_{D}$ and, in particular, the global results (e.g., the all-particle spectrum and the average primary mass as a function of primary energy) are roughly independent from it. This can be seen in Fig. 18 where the average mass is plotted against primary

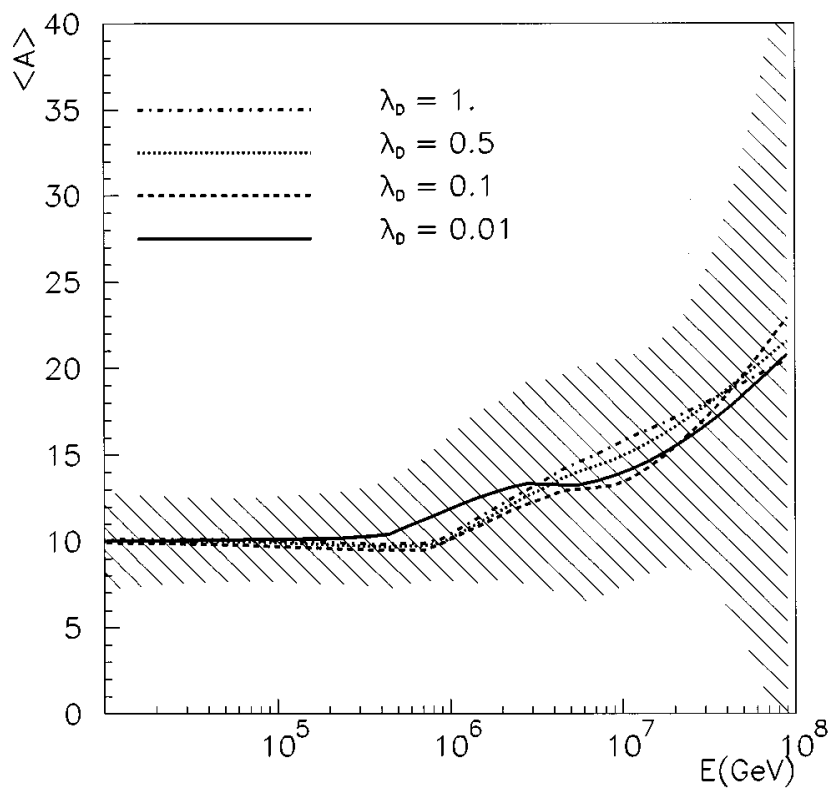

FIG. 18. Average mass as a function of primary energy at different values of the direct measurement weight parameter $\left(\lambda_{D}\right)$. The hatched area gives $\langle A\rangle$ within $\pm 1 \sigma$ error for $\lambda_{D}=0.01$. energy at various values of $\lambda_{D}$. This fact gives us confidence in the estimate of the flux parameters and points out the high potential of multimuon data in the determination of the primary composition.

The actual value of the limiting minimum of $\xi^{2}$ $\left(\xi_{\min }^{2}=13.6\right)$, which appears to be somewhat lower than the "equivalent" number of degrees of freedom $[\nu=24$, as given by Eq. (B4)], requires additional discussion. The corresponding confidence level turns out to be $\sim 95 \%$ and could suggest an overestimate of the error in the multimuon rates at the various multiplicities [see Eq. (7)]. The various sources of experimental errors on muon rates are discussed in [21]. These include errors from muon reconstruction (formula (5.1) in [21]), unfolding errors in the high multiplicity region and systematic errors to account for the uncertainties in the merging of the reconstructed data sample with the scanned one. Apart from muon multiplicities $\leqslant 15$, where $\sigma\left[R^{\text {meas }}\left(N_{\mu}\right)\right]$ is roughly equal to the statistical error at higher multiplicities both the systematic errors, and the unfolding errors are rather large and higher than the equivalent statistical ones. This fact, which is inherent in the adopted method of analysis, partly explains the intrinsically low value of $\xi_{\min }^{2} / N_{\mathrm{DF}}$. Furthermore, we recall that also the errors on predicted rates [see Eq. (7)] are roughly of the same order as the experimental ones. This is due to the impossibility of increasing the size of the simulated data sample on account of the extremely long CPU time required in the event generation (see Sec. II).

\section{APPENDIX D: BEST FIT PARAMETERS AND ERRORS}

In the previous Appendix, we showed that the constrained fit reaches an asymptotic value of $\xi_{\min }^{2}$ at $\lambda_{D} \leqslant 0.01$. In Sec. III B we give our results at $\lambda_{D}=0.01$ for which the convergence of the minimization process is reached without problem and the covariance matrix is still accurate. The best fit parameters obtained at this $\lambda_{D}$ value, for the case of $\chi_{M}^{2}$ built up with the absolute muon rates $R\left(N_{\mu}\right),(A / R$ fit) are shown in Table IV, in the usual ultrahigh energy (UHE) cosmic ray (CR) energy scale $(\mathrm{GeV})$. Parameter errors are not given in that table, since they are meaningless at such a scale, so different from the one actually used in our fit (see Appendix A), because of the strong correlations among parameters. Table VII shows the best fit parameters obtained in the $A / R$ fit, from which the values reported in Table IV are derived. As it can be easily recognized, both the energy scale and the redefinition of the parameters render the minimization free of possible numerical inaccuracies, being the best fit parameters roughly of the same size. Furthermore, the covariance matrix is accurate since also its elements range in a rather small interval of possible values. This is shown in Table VIII, where the nondiagonal elements of the correlation matrix are shown.

\section{APPENDIX E: CONSISTENCY CHECK OF THE MINIMIZATION PROCEDURE}

In order to check our minimization procedure, we tried to reconstruct a priori known primary cosmic ray composi- 
TABLE VII. Best fit parameters as obtained from the fit of the absolute muon rates $(A / R$ fit). For comparison the parameters obtained from the fit of MACRO data alone ( $M / A$ fit) and the fit of direct measurements (see Sec. III B) are also given. The parameters of the $M / A$ fit are reported without errors being obtained from a fit with boundary conditions (see Sec. III B and Appendix A). $\kappa_{1}$ parameters are expressed in $\mathrm{m}^{-2} \mathrm{~s}^{-1} \mathrm{sr}^{-1}(10 \mathrm{TeV})^{-1}$.

\begin{tabular}{ccccc}
\hline \hline & & MACRO & MACRO & Direct \\
No. & Type & $A / R$ fit & $M / A$ fit & measurements fit \\
\hline 1 & $\gamma_{1}(\mathrm{H})$ & $2.67 \pm 0.13$ & 2.7 & $2.81 \pm 0.04$ \\
2 & $\gamma_{1}(\mathrm{He})$ & $2.47 \pm 0.21$ & 2.4 & $2.65 \pm 0.07$ \\
3 & $\gamma_{1}(\mathrm{CNO})$ & $2.42 \pm 0.15$ & 2.4 & $2.56 \pm 0.03$ \\
4 & $\gamma_{1}(\mathrm{Mg})$ & $2.48 \pm 0.12$ & 2.4 & $2.57 \pm 0.03$ \\
5 & $\gamma_{1}(\mathrm{Fe})$ & $2.67 \pm 0.16$ & 2.7 & $2.61 \pm 0.06$ \\
6 & $\gamma_{2}(\mathrm{H})$ & $2.78 \pm 0.13$ & 2.8 & \\
7 & $\gamma_{2}(\mathrm{He})$ & $3.13 \pm 0.26$ & 3.1 & \\
8 & $\gamma_{2}(\mathrm{CNO})$ & $3.58 \pm 0.53$ & 3.2 & \\
9 & $\gamma_{2}(\mathrm{Mg})$ & $3.31 \pm 0.53$ & 3.2 & \\
10 & $\gamma_{2}(\mathrm{Fe})$ & $2.46 \pm 0.72$ & 2.8 & \\
11 & $\kappa_{1}(\mathrm{H})$ & $2.55 \pm 0.24$ & 2.6 & $1.99 \pm 0.08$ \\
12 & $\kappa_{1}(\mathrm{He})$ & $1.7 \pm 1.1$ & 1.4 & $1.75 \pm 0.17$ \\
13 & $\kappa_{1}(\mathrm{CNO})$ & $0.81 \pm 0.31$ & 0.90 & $0.72 \pm 0.03$ \\
14 & $\kappa_{1}(\mathrm{Mg})$ & $0.53 \pm 0.18$ & 0.61 & $0.49 \pm 0.02$ \\
15 & $\kappa_{1}(\mathrm{Fe})$ & $0.50 \pm 0.21$ & 0.63 & $0.52 \pm 0.03$ \\
16 & $\Lambda_{\text {cut }}(\mathrm{Fe})$ & $2.75 \pm 0.26$ & 02.5 & \\
\hline \hline
\end{tabular}

tions. For this purpose events generated using specific test composition models have been processed through the same multiparametric fit procedure as experimental data and the fitted spectra have been compared with the input ones. Figure 19 shows the result for one of these checks when the test composition model is a constant mass composition with rigidity dependent cutoff. The elemental spectra are determined by only eight parameters: five normalization factors
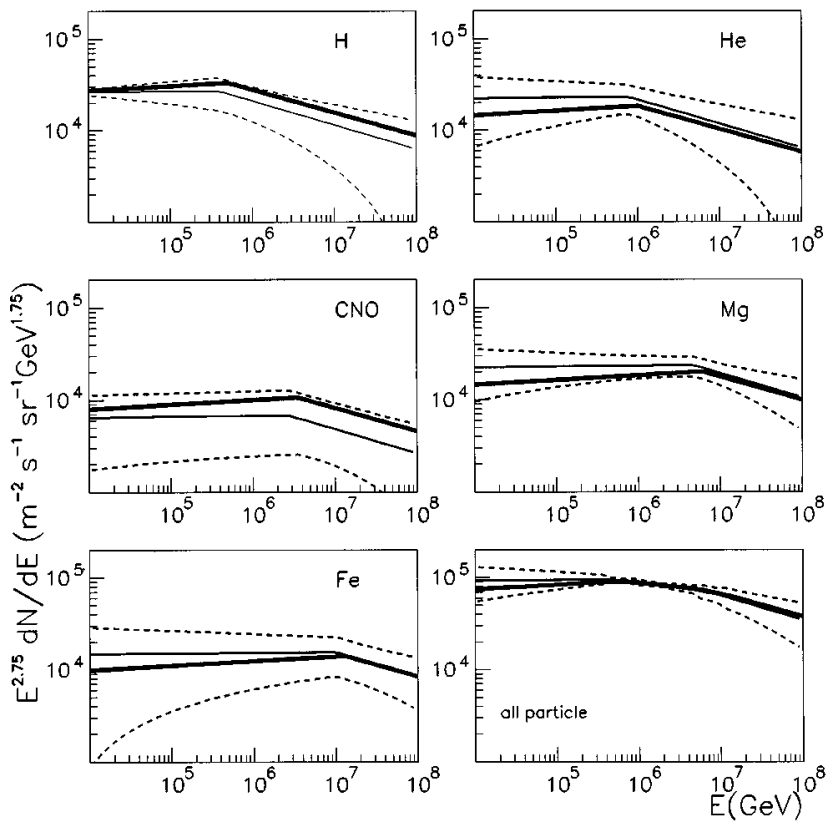

FIG. 19. Input spectra (bold line) and reconstructed spectra for a constant mass composition model with spectral indexes $\gamma_{1}=2.7$ and $\gamma_{2}=3$ and a rigidity cutoff of $500 \mathrm{TV}$.

$K_{1}(A)$, two spectral indexes $\gamma_{1}$ and $\gamma_{2}$, equal for each group, and one energy cutoff. This case is of particular interest for demonstrating the sensitivity of MACRO data to composition, since it allows us to perform the minimization of the $\chi_{M}^{2}$ term alone [i.e., setting $\lambda_{D}=0$ in Eq. (9)], without the need of the constraining term $\lambda_{D} \cdot \chi_{D}^{2}$ and the consequent extrapolation procedure described in Appendix C. It is important to point out that the comparison between input spectra and fitted ones has to be done on their energy dependence and not comparing individual parameters. Each estimated parameter is expected to agree with the corresponding input

TABLE VIII. Nondiagonal correlation matrix elements as obtained from the $A / R$ fit. The correspondence between parameter number and type is given in Table VII.

\begin{tabular}{|c|c|c|c|c|c|c|c|c|c|c|c|c|c|c|c|}
\hline No. & 1 & 2 & 3 & 4 & 5 & 6 & 7 & 8 & 9 & 10 & 11 & 12 & 13 & 14 & 15 \\
\hline 2 & -0.37 & & & & & & & & & & & & & & \\
\hline 3 & 0.33 & -0.42 & & & & & & & & & & & & & \\
\hline 4 & -0.01 & 0.01 & -0.02 & & & & & & & & & & & & \\
\hline 5 & 0.00 & -0.06 & 0.14 & 0.00 & & & & & & & & & & & \\
\hline 6 & -0.41 & -0.02 & -0.03 & -0.09 & -0.34 & & & & & & & & & & \\
\hline 7 & 0.45 & -0.38 & 0.42 & 0.05 & 0.01 & -0.22 & & & & & & & & & \\
\hline 8 & -0.21 & 0.17 & 0.12 & 0.03 & -0.37 & 0.32 & -0.17 & & & & & & & & \\
\hline 9 & 0.09 & -0.16 & 0.40 & -0.43 & -0.24 & 0.19 & 0.33 & 0.03 & & & & & & & \\
\hline 10 & 0.02 & 0.09 & -0.31 & 0.02 & -0.84 & 0.23 & -0.08 & 0.15 & 0.08 & & & & & & \\
\hline 11 & 0.39 & -0.93 & 0.24 & 0.03 & 0.03 & -0.06 & 0.27 & -0.12 & 0.05 & -0.03 & & & & & \\
\hline 12 & 0.08 & 0.87 & -0.18 & 0.00 & -0.03 & -0.19 & -0.12 & 0.08 & -0.06 & 0.04 & -0.87 & & & & \\
\hline 13 & -0.02 & 0.10 & 0.43 & 0.00 & -0.01 & -0.01 & -0.07 & -0.01 & -0.03 & 0.03 & -0.07 & 0.03 & & & \\
\hline 14 & 0.00 & 0.03 & 0.00 & 0.22 & -0.01 & 0.03 & -0.04 & -0.03 & 0.13 & 0.01 & -0.03 & 0.01 & 0.00 & & \\
\hline 15 & 0.01 & 0.01 & -0.03 & 0.01 & 0.13 & 0.11 & 0.00 & 0.12 & 0.07 & 0.26 & -0.01 & 0.01 & 0.00 & 0.00 & \\
\hline 16 & 0.17 & -0.32 & 0.70 & 0.39 & 0.17 & 0.03 & 0.53 & 0.29 & 0.20 & -0.38 & 0.18 & -0.13 & -0.06 & -0.14 & -0.04 \\
\hline
\end{tabular}


parameter within $\pm 1 \sigma$, at $68 \%$ C.L., when all the others have any possible value in the parameter space [52]. Having in mind this caveat, the consistency check turns out to be successful. The agreement between the spectra obtained from the fitting procedure and the input spectra (Fig. 19) confirms the ability of the multiparametric fit procedure to reconstruct the primary energy spectra.

\section{ACKNOWLEDGMENTS}

We would like to thank F. James, D. Drijard, and E. Di Capua for suggestions given in the function minimization and error handling. We also thank T. K. Gaisser and T. Stanev for many useful discussions about hadronic interaction models.
[1] P. O. Lagage and C. J. Cesarsky, Astron. Astrophys. 125, 249 (1983).

[2] For a review of acceleration mechanisms and diffusive propagation see, for example, T. K. Gaisser, Cosmic Rays and Particle Physics (Cambridge University Press, Cambridge, England, 1990).

[3] H. J. Völk and P. L. Biermann, Astrophys. J. Lett. 333, L65 (1988).

[4] G. V. Kulikov and G. B. Khristiansen, Sov. Phys. JETP 35, 635 (1958).

[5] T.V. Danilova et al., Proceedings of 15th International Cosmic Ray Conference, Plovdiv, Bulgaria, 1977 (Bulgaria Academy of Sciences, Plovdiv, 1977), Vol. 8, p. 129.

[6] M. Nagano et al., J. Phys. G 10, 1295 (1984).

[7] Yu. Fomin et al., in Proceedings of the 22nd International Cosmic Ray Conference, Dublin, Ireland, 1991, edited by M. Cawley et al. (Dublin Institute for Advanced Studies, Dublin, 1992), Vol. 2, p. 85.

[8] BASJE Collaboration, H. Yoshii et al., in Proceedings of the 24th International Cosmic Ray Conference, Rome, Italy, 1995, edited by N. Iucci et al. (Arti Grafiche Editoriali Srl, Urbino, 1995), Vol. 2, p. 703; Y. Shirasaki, Master thesis, Tokyo Institute of Technology, 1994.

[9] S. V. Bryanski et al., in Proceedings of the 24th International Cosmic Ray Conference [8], Vol. 2, p. 724.

[10] Tibet AS $\gamma$ Collaboration, M. Amenomori et al., in Proceedings of the 24th International Cosmic Ray Conference [8], Vol. 2, p. 736; ICRR Report No. 349-95-15 1995 (unpublished).

[11] A. E. Chudakov et al., in Proceedings of the 22nd International Cosmic Ray Conference [7], Vol. 2, p. 5; V. N. Bakatanov et al., in Proceedings of the 24th International Cosmic Ray Conference [8], Vol. 2, p. 720.

[12] D. B. Kieda, Ph.D. thesis, University of Utah, 1989.

[13] G. Bologna et al., Nuovo Cimento C 8, 76 (1985); M. Aglietta et al., in Proceedings of the Topical Seminar on Astrophysics and Particle Physics, San Miniato, Italy, 1989, edited by F. L. Navarria and P. G. Pelfer [Nucl. Phys. B (Proc. Suppl.) 14, 193 (1990)].

[14] W. W. M. Allison et al., in Proceedings of the 23rd International Cosmic Ray Conference, Calgary, Canada, 1993, edited by R. B. Hicks et al. (World Scientific, Singapore, 1994), Vol. 4, p. 398; S. M. Kasahara, Ph.D. thesis, University of Minnesota, 1995 (unpublished).

[15] MACRO Collaboration, S. P. Ahlen et al., Phys. Rev. D 46, 895 (1992).

[16] MACRO Collaboration, S. P. Ahlen et al., in Proceedings of the 23rd International Cosmic Ray Conference [14], Vol. 2, p. 97.

[17] MACRO Collaboration, S. P. Ahlen et al., "Underground muon physics at MACRO,' Report No. LNGS-94/114, 1994 (unpublished).

[18] G. Auriemma et al., in Proceedings of the 21st International Cosmic Ray Conference, Adelaide, Australia, 1990, edited by R. J. Protheroe (Graphic Services, Northfield, South Australia, 1990), Vol. 9, p. 362.

[19] J. Kempa and J. Wdowczyk, J. Phys. G 9, 1271 (1983).

[20] EASTOP and MACRO Collaborations, M. Aglietta et al., Phys. Lett. B 337, 376 (1994).

[21] MACRO Collaboration, M. Ambrosio et al., preceding paper, Phys. Rev. D 56, 1407 (1997).

[22] R. Brun et al., CERN Report No. CERN DD/EE/84-1, 1992 (unpublished).

[23] C. Forti et al., Phys. Rev. D 42, 3668 (1990).

[24] G. J. Alner et al., Phys. Lett. 167B, 476 (1986).

[25] MACRO Collaboration, S. P. Ahlen et al., Phys. Rev. D 46, 4863 (1992).

[26] MACRO Collaboration, S. P. Ahlen et al., in Proceedings of the 23rd International Cosmic Ray Conference [14], Vol. 2, p. 93.

[27] J. Engel et al., Phys. Rev. D 46, 5013 (1992).

[28] J. W. Elbert, T. K. Gaisser, and T. Stanev, Phys. Rev. D 27, 1448 (1983).

[29] G. Battistoni et al., Astropart. Phys. 4, 351 (1996).

[30] G. Battistoni, C. Forti, and J. Ranft, Astropart. Phys. 3, 157 (1995).

[31] R. S. Fletcher et al., Phys. Rev. D 50, 5710 (1994).

[32] See for instance A. Capella et al., Phys. Rev. Lett. 58, 2015 (1987), and references therein.

[33] T. K. Gaisser and T. Stanev, Phys. Lett. B 219, 375 (1989).

[34] T. K. Gaisser, Proceedings of Frontier Objects in Astrophysics and Particle Physics, Vulcano, Italy, 1992 (Editrice Compositon. Bologna, 1993), p. 433.

[35] J. Ranft, Phys. Rev. D 51, 64 (1995).

[36] J. Knapp, D. Heck, and G. Schatz, Forschungszentrum Karlsruhe Report No. FZKA 5828, 1996 (unpublished).

[37] MACRO Collaboration, M. Ambrosio et al., Phys. Rev. D 52, 3793 (1995).

[38] H. Bilokon et al., "Muon survival probabilities in the Gran Sasso rock,' Report No. LNGS-94/92, 1994 (unpublished).

[39] P. Lipari and T. Stanev, Phys. Rev. D 44, 3543 (1991).

[40] A. Fassó et al., FLUKA: Present Status and Future Developments, in Proceedings of the IV International Conference on Calorimetry in High Energy Physics, La Biodola, Italy, 1993 (World Scientific, Singapore, 1993), p. 493.

[41] F. James, “MINUIT reference manual,' CERN, 1994 (unpublished).

[42] B. Peters, Proceedings of the 6th International Cosmic Ray Conference, Moscow, USSR, 1959 (Moscow, 1960), Vol. 3, 
p. 157; Nuovo Cimento 22, 800 (1961).

[43] JACEE Collaboration, T. H. Burnett et al., Astrophys. J. 349, L25 (1990); in Proceedings of the 23rd International Cosmic Ray Conference [14], Vol. 2, pp. 5, 21, and 25.

[44] I.P Ivanenko et al., in Proceedings of the 22nd International Cosmic Ray Conference [7], Vol. 2, p. 17.

[45] CRN Collaboration, D. Müller et al., Astrophys. J. 374, 356 (1991).

[46] B. Wiebel-Sooth et al., in Proceedings of the 24th International Cosmic Ray Conference [8], Vol. 2, p. 656; B. Wiebel, University of Wuppertal, Report No. WUB 94-08, 1994 (unpublished).

[47] N. L. Grigorov et al., in Proceedings of the 12th International Cosmic Ray Conference, Hobart, Tasmania, 1971, edited by A.
G. Fenton and K. B. Fenton (University of Tasmania Press, Hobart, Tasmania, 1971), Vol. 5, p. 1746.

[48] T. V. Danilova, A. D. Erlykin, and J. Procureur, J. Phys. G 19, 429 (1993).

[49] P. Stavrev, N. Gavritova-Stavreva, and S. Petrov, J. Phys. G 18, 1833 (1992).

[50] D. J. Bird et al., Astrophys. J. 424, 491 (1994); P. Sokolsky, in Proceedings of the 23rd International Cosmic Ray Conference [14], p. 447.

[51] S.P. Swordy, in Proceedings of the 24th International Cosmic Ray Conference [8], Vol. 2, p. 697.

[52] F. James, 'Function Minimization,' Report No. CERN 72-21, 1972 (unpublished). 